\title{
The Use of Molecular Dynamics for the Thermodynarnic Properties of Simple and Transition Metals
}

\author{
Galen K. Straub
}

\author{
DISCLAIMER
}

This report was prepared as an account of work sponsored by an agency of the United States This repment. Neither the United States Government nor any agency thereof, nor any of their employees, makes any warranty, express or implied, or assumes any legal liability or responsi垈, completeness, or usefulness of any information, apparatus, product, or bility for the accuracy, complet that its use would not infringe privately owned rights. Referprocess disclosed, or represents that its use wout process, or service by trade name, trademark, ence herein to any specific commercial product, process, or se imply its endorsement, recommanufacturer, or otherwise does not necessarily constient or any agency thereof. The views mendation, or favoring by the United States Government or any age or reflect those of the

and opinions of authors expressed herein do not

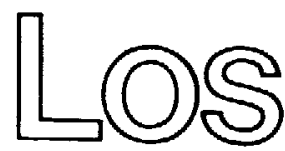


THE USE OF MOLECULAR DYNAMICS FOR THE THERMODYNAMIC PROPERTIES OF SIMPLE AND TRANSITION METALS

by

Galen K. Straub

ABSTRACT

The technique of computer simulation of the molecular dynamics in metallic systems to calculate thermodynamic properties is discussed. The nature of a metal as determined by its electronic structure is used to determine the total adiabatic potential. The effective screened ion-ion interaction can then be used in a molecular dynamics simuiation. The method for the construction of a molecular dynamics ensemble, its relation to the canonical ensemble, and the definition of thermodynamic functions from the Helmholtz free energy is given. The method for the analysis of the molecular dynamics results from quasiharmonic Iattice dynamics and the decomposition in terms of harmonic and anharmonic contributions is given for solids. For fluid phase metals, procedures for calculating the thernodynamics and determining the constant of entropy are presented. The solid-fluid phase boundary as a function of pressure and temperature is determined using the results of molecular dynamics. Throughout, examples and results for metallic sodium are used. The treatment of the transition metal electronic d-states in terms of an effective pair-wise interaction is also discussed and the phonon dispersion curves of $\mathrm{Al}, \mathrm{Ni}$, and $\mathrm{Cu}$ are calculated. 


\section{INTRODUCTION}

Computer simulation offers a very powerful technique for highly accurate calculations of the thermodynamic functions in metallic systems. We will present some of the reasons for using molecular dynamics (herein abbreviated as MD) and methodology for studying the thermodynamic properties of metals. This will include discussions on the nature of a metallic system, statistical mechanics, the construction of ensembles from MD, and the definitions of thermodynamic functions. MD is not the only way one can obtain the thermodynamic properties of a metal, but at high temperatures in the solid and in the fluid phase, MD allows a straightforward, highly accurate solution. I will try to elucidate the properties of a MD system and how the fact that we are in a metal has a dominating effect on the procedures that we use. The path to thermodynamics is by way of statistical mechanics. This means that we must know how to take our MD results and interpret them as a MD ensemble. Following that, we must establish the link between a MD ensemble and a canonical ensemble, and finally, the definitions of thermodynamics in terms of a canonical ensemble.

A MD simulation consists of a system of $\mathrm{N}$ particles interacting with one another via some force law in a computational cell, a set of initial positions and velocities, a set of boundary conditions on the computational cell, and the time integrated evolution of the equations of motion. I shall consider only the case of periodic boundary conditions and fixed volume for the computational cell. The total energy is assumed to be a constant of the motion. The primary product of the MD simulation is a set of positions $\{r(t)\}$ and a set of velocities $\{v(t)\}$ as a function of time. The MD system is a mechanical one that solves for the time evolution of the classical equations-of-motion. We can use the sets of positions and velocities to construct other mechanical quantities such as the total kinetic energy and the total potential energy. In Fig. 1 , we give the total kinetic energy as a function of time for two different $M D$ calculations.

Both of the calculations shown in Fig. 1 consisted of about 1000 particles whose initial positions were those of a bcc lattice. The initial velocities were chosen randomly and with a different amount of total energy for the two calculations. The curve labeled "fluid" is for a MD system whose final state was in the fluid phase and the curve labeled "solid" had a final state in the 


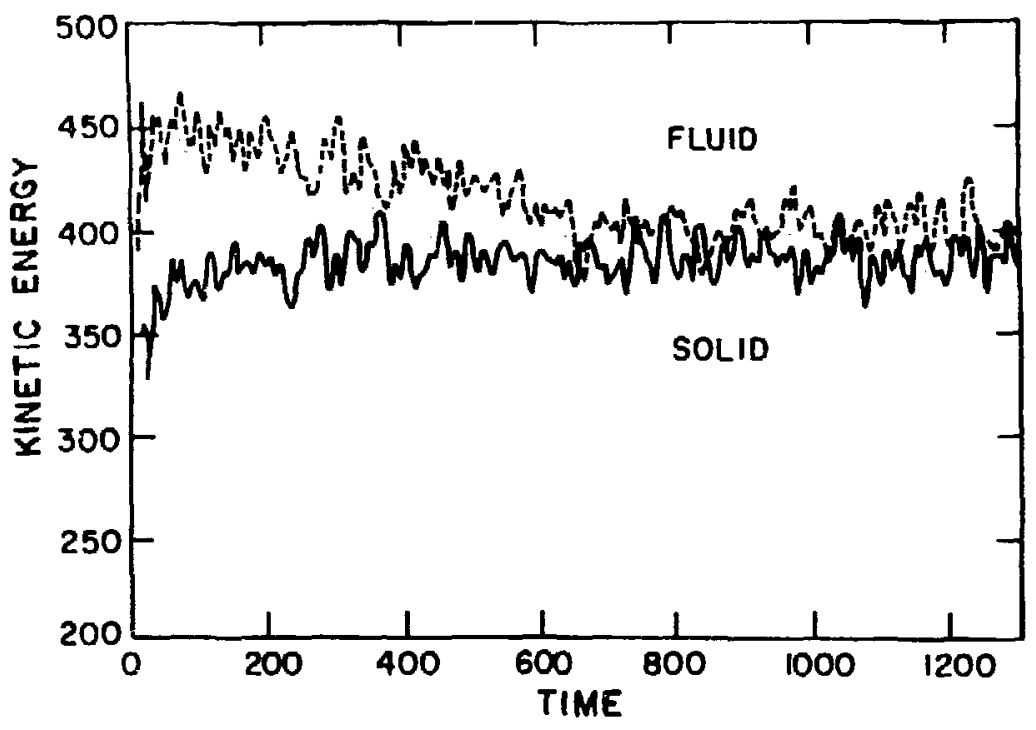

Fig. 1.

KINETIC ENERGY VS. TIME FOR A MD CALCULATION. The curve labeled "fluid" is for a MD system whose final state was in the fluid phase and the curve labeled "solid" had a final state in the solid phase. Both calculations were started in a bcc lattice with about 1000 particles, but with different initial kinetic energies. The solid system reaches equilibrium around Time-500, while the fluid calculation does not reach equilibrium until Time-700. Averaging for thermodynamic quantities must wait until the system is in equilibrium.

solid phase. We start the calculation at Time $=0$ and wait for the system to come to mechanical equilibrium. The solid system reaches equilibrium around Time $=500$ (arbitrary units), while the fluid calculation does not reach equilibrium until Time=700. These two curves represent the type of basic information that one gets directly from MD. The first step is to construct a suitable ensemble to do statistical mechanics; we shall call this ensemble the MD ensemble. In order to construct statistical ensembles we must wait until the system is in mechanical equilibrium and then form the appropriate averages. The procedure for choosing the start time for averaging will be discussed in more detail below.

In statistical mechanics, an ensemble is a very large number of systems in contact with the appropriate reservoir and with constraints for each system. We will be concerned with two different ensembles: (1) the MD ensemble that we will construct from time averages of dynamical variables such as shown in Fig. 1 ; and, (2) the canonical ensemble that provides the direct link to defining thermodynamics from statistical mechanics. The canonical ensemble constrains 
each system to a constant number of particles $N$ at fixed volume $V$ in contact with a reservoir at constant temperature $T$. The MD ensemble consists of a large number of systems having constant $\mathrm{N}$ and $\mathrm{V}$ with the total energy held constant. The reservoir for each MD system is an angular momentum reservoir. The MD ensemble is not a microcanonical ensemble, since the microcanonical ensemble has no restriction on the total angular momentum.

We want to define thermodynamic functions in the MD ensemble in terms of averages of the dynamic variables as one does for the canonical ensemble, but here we run into a problem. The averages of the same dynamical variable will be different in different ensembles. For quantities that only involve averages of dynamical variables, the resulting difference between ensembles is of relative $\operatorname{order} 1 / \mathrm{N}$. Thus, for instance, we can calculate the temperature $\mathrm{T}$ from a $\mathrm{MD}$ ensemble by averaging the kinetic energy, but this average will differ from averages of a canonical ensemble by

$<$ K.E. $\rangle_{M D}=\langle\text { K.E. }\rangle_{\text {canonical }}+O(1 / N)$,

where $O(1 / N)$ signifies terms of relative order $1 / N$. This poses no problem at all since we can easily make $\mathrm{N}-10^{3}$. On the other hand, if we are interested in calculating averages of the fluctuation of a dynamical variable, then the corrections are of $O(1)$. The calculation of the elastic constants involves averages over the fluctuations of the interparticle potential energy and correcting the MD averages to the canonical ensemble averages is essential. These last statements may be summarized by the following: let $A \equiv$ dynamical variable, $\langle A\rangle$ average, and the fluctuation of $A, \delta A \equiv A-\langle A\rangle$, then

$\langle A\rangle_{M D}=\langle A\rangle_{\text {canonical }}+O(1 / N)$,

$\langle\delta A\rangle_{M D}=\langle\delta A\rangle_{\text {canonical }}+O(1)$.

For the present discussion, we will not have to deal with averages of fluctuations. Formulas for the corrections of fluctuations between MD and canonical ensembles have been derived in detail in Refs. (1) and (2). The calculation of the elastic constants from the MD ensemble and the ensemble corrections are given in Refs. (3) and (4).

In metals, the net force on any particular ion is the result of screened long range coulomb interactions. A metal consists of a coupled system of conduction electrons and charged ions. The conduction electrons may be considered to remain in their ground state and are responsible for the screening of the ionic coulomb potential. The Born-Oppenheimer adiabatic approximation 
may be used to determine the ion-ion interaction. (5) The adiabatic approximation allows us to solve the electronic structure problem for the ions at some set of fixed positions. The positions are arbitrary, but fixed. The adiabatic potential is the sum of the ion-ion interaction plus the electronic energy where both are functions of the ionic positions. The vibrational energy of the ions may then be solved using the adiabatic potential. The conduction electrons contribute in two ways. First, as an electron gas their kinetic, exchange, and correlation energy may be written as a function of volume only. Second, they act to screen the ions from one another and reduce the range of the the coulomb forces. A useful way of looking at the forces between ions is that the screened interaction is a direct function of the electron density. As a result, the forces between a system of ions are an explicit function of the volume.

The problem to be addressed for the simulation of a metal by MD is to accurately calculate the ribrational energies of the ions, which means that we must properly represent the net many-body forces between the ions for integration of the equations-of-motion. The adiabatic approximation allows us to write the potential energy as the sum of two terms, a volume dependent term resulting mainly from the conduction electrons, and a many-body ion term that is an explicit function of volume. The result of using the many-body ion term alone would be an unstable system since in general neighboring ions do not lie at the minimum of the ion-ion potential. Including the volume dependent terms, the system is stable and lies at the minimum of the total adiabatic potential well. In MD, we can use the periodic boundary conditions to confine the system to a fixed volume. Both terms are essential for the description of a metallic material. Because of the importance of the volume dependence in the potential errergy, Section II is devoted to a more detailed discussion of this point and Section III discusses the treatment of the transition metals.

The procedure for applying statistical mechanics to a MD simulation giving the route from a mechanical theory to a thermodynamic one is given in Section IV. This involves some formal definitions of thermodynamic quantities in terms of statistical averages as mentioned above. In Section $v$, the calculation of the total energy and pressure from $M$ will be given. Then, the analysis of these quantities will be given whereby one may construct the Helmholtz fres energy throughout phase space. In the solid we make use of the theory of 
lattice dynamics $(6)$ and this topic is presented in section VI. Lattice dynamics gives the correct answers for thermodynamics in the low temperature quantum mechanical regime where $M D$, because of its restriction to classical equations-of-motion, fails. Below the Debye temperature $\theta_{0}(\mathrm{Al}=428 \mathrm{~K}, \mathrm{Cu}=345 \mathrm{~K}$, $\mathrm{Na}=153 \mathrm{~K}$ ) quantum mechanical effects may not be neglected as illustrated by experimental data for the heat capacity. Above $\theta_{0}$, vibrational anharmonicity becomes important and the decomposition of the energy and pressure into harmonic and anharmonic contributions will be given. For the fluid phase, the classical equations of MD are adequate although the electron gas energy requires a high temperature correction. Unfortunately, one does not have a reference theory, such as harmonic lattice dynamics for the solid, and the fluid phase thermodynamics must be treated using numerical fits of the MD results.

Another difficulty arises in the treatment of thermodynamics of the fluid phase, namely, the determination of the constant in the entropy. In a classical description the value of the entropy is not unique, but only defined to within an arbitrary constant. This problem is handled in section $\mathrm{V}$ by making a high temperature cluster expansion of the free energy (which contains the entropy constant) and evaluating the free energy at one point in phase space with the aid of MD. The free energy can ther be integrated down in temperature to the region of interest, in our case so that we may construct the solid-fluid phase boundary. A problem with the constant of entropy for the solid does not arise since we treat the solid phase as a quantur mechanical system at low temperatures with $M D$ as an approximate description appropriate only above the Debye temperature. In quantum mechanics, the constant of the entropy may be uniquely determined.

Throughout the text, the calculational methods will be illustrated for the example of solid and fluid metallic sodium. The same methods may be applied to transition metals, but the effective ion-ion interaction needed for MD is more complicated. Section III gives some details of the electronic structure and effective ionic potentials for transition metals.

II. TOTAL ADIABATIC POTENTIAL FOR A METAL

The Born-Oppenheimer adiabatic approximation for a coupled system of electrons and ions allows us to write the potential energy of a metal in terms 
of the ion coordinates, but with the electron coordinates as explicit parameters. (5) This decomposition is possible because the vibrational motion of the ions has a characteristic time scale of the order of $10^{-14} \mathrm{sec}$. while electronic time scales are of the order of $10^{-17}$ to $10^{-18} \mathrm{sec}$. In terms of a physical picture, this means that for any configuration or change in configuration of the ions, the electrons respond so quickly that they remain in an energy ground state. The term "adiabatic" refers to this situation.

We take as our model a system of $\mathrm{N}$ ions and $\mathrm{NZ}$ electrons in a volume $\mathrm{V}$. In the adiabatic approximation, the total energy is the sum of the kinetic energies of ail the ions, plus the total adiabatic potential $\Phi$. We pick the zero of $\Phi$ to correspond to having neutral atoms infinitely separated and $I_{Z}$ is the ionization energy. $\Phi$ consists of an electron gas term $E_{G}$, the ion-ion coulomb interaction $\Omega_{i-i}$, the electron-ion interaction $\Omega_{e-i}$, and the electron-electron interaction $\Omega_{e-e}$. The electron-ion and electron-electron terms are combined in the framework of pseudopotential theory with the result (7), (8)

$$
\Phi=N I_{z}+\Omega(V)+\sum \phi(r ; V) .
$$

$\Omega(V)$ is a volume-dependent term and $\sum \phi(r ; V)$ is the summation over the many-body effective ion-ion interaction. To this point, there is no restriction to two-body interactions with the only approximation being the use of perturbation theory to solve for the electron energies. In practice, we truncate the perturbation expansion at second order, rearrange terms and write $\phi$ as an effective two-body interaction between ions that includes the effects of the electron-ion interactions and electron-electron screening interactions. (Third order in the perturbation expansion gives three-body interactions, etc.) In any case, $\phi(r ; V)$ is an explicit function of the volume as well as the interparticle separation $r$.

Fig. 2 is a plot of the total effective pair potential for Na calculated for the point-ion pseudopotential. The volume dependence of the pair interaction is clearly apparent. At the zero-pressure volume in a bcc lattice, the first neighbors lie on the repulsive side of the minimum and the second neighbors on the attractive side. $\phi$ is also very long range and oscillates about zero (thev are not visible on the scale of Fig. 2; see Refs. (9) and (10)). These long range oscillations are the result of the electron screening and are called Friedel oscillations. We need not be concerned with them in the present discussion, jut in practice, a total of ten shells of neighbors $(-200)$ was 


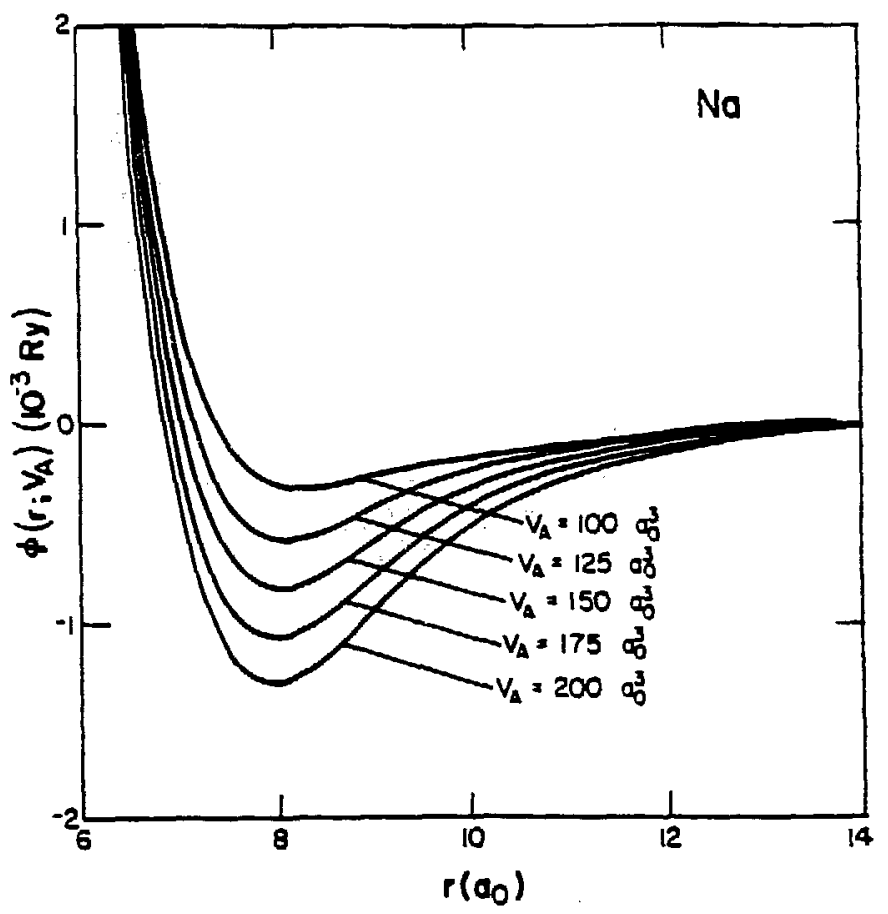

Fig. 2.

TOTAL EFFECTIVE PAIR POTENTIAL FOR Na CALCULATED FROM THE MODIFIED POINT-ION PSEUDOPOTENTIAL. Our system consists of $\mathrm{N}$ ions and $\mathrm{NZ}$ electrons in a volume $\mathrm{V}$, and is described by an adiabatic potential $\Phi$ evaluated in the pseudopotential perturbation formulation. $\Phi$ consists of two parts: a volume-dependent term $\Omega(V)$, and a sum over all distinct pairs of ions of the effective ion-ion potential $\phi(r ; V) . \phi$ includes the effects of the electron-ion interactions and the many body electron-electron screening interactions. The potential energy is in units of rydbergs (Ry) and the separation is in units of Bohr radil ( $\left.a_{0}\right)$.

routinely included in $\sum \phi$.

The contributions to the total adiabatic potential $\Phi$ as a function of volume are plotted in Fig. 3. Nearly $90 \%$ of the total potential energy comes from the volume dependent terms in $\Omega$ while only 108 comes from the $\sum \phi(r ; V)$. The energy is a minimum at the experimental value of the volume at zero pressure. Thus, to first order in the pseudopotential perturbation expansion there is no need for a pair-potential at all (we still need a nseudopotential since in first order it is an important contribution in the volume dependent terms) and quite reasonable estimates and trends in simple and transition metals may be made.(8),(11) However, for thermodynamic properties as a function of temperature we require the vibrational properties of the ions, and this information is contained in $\sum \phi(r ; V)$ where the summation is over the sets of positions of the ions $(r(t))$. 

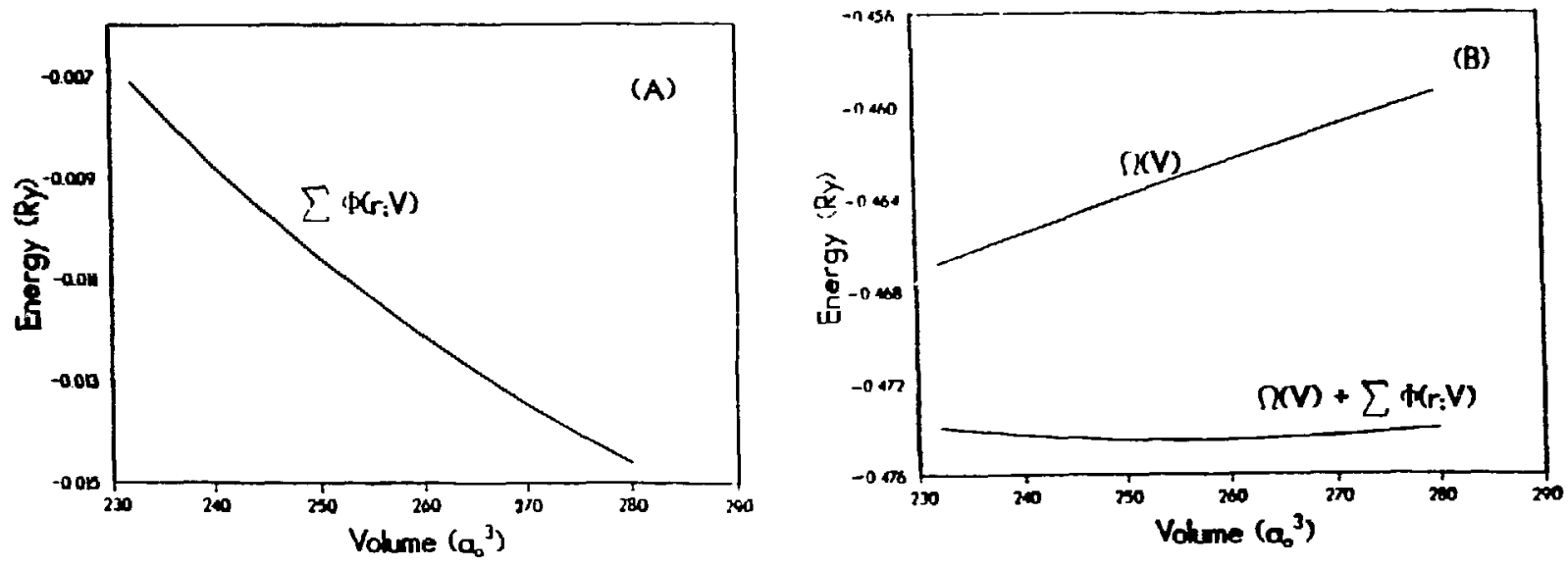

Fig. 3.

THE CONTRIBUTIONS TO THE TOTAL ADIABATIC POTENTIAL $\Phi$ AS A FUNCTION OF VOLUME.

(A) The quantity $\sum \phi(r ; V)$ as a function of volume ( $a_{0}=B o h r$ radius $=0.529 \dot{A}$ ). The volume dependent term $\Omega(V)$ and the total adiabatic potential $\Phi-N I_{z}=\Omega(V)+$ $\sum \phi(r ; V)$. Note the large difference in scales of panels (A) and (B). Nearly 908 of the total energy comes from the volume dependent terms alone. The energy is a minimum at the experimental value of the volume at zero pressure.

\section{ELECTRONIC STRUCTURE AND EFFECTIVE IONIC POTENTIALS IN TRANSITION METALS}

For transition metals, Eq. (II.1) holds as it is written except now $\phi$ contains terms that describe the $d-d$ and $d$-conduction electron interactions. At first thought, one might expect that the addition of the d-electrons might make such an approach much too complicated. In fact, the treatment of the transition metals is as straightforward as for the simple metals. This is possible because the cohesive energy in transition metals is primarily due to the presence of the d-electrons. One of the most complex aspects of the treatment of the simple metals is the electron screening among the conduction electrons. Because of its reduced relative importance in the transition metals, we may further approximate this screening to simplify the theory but without a great overa11 loss in accuracy.

The presence of the d-electrons in the transition metals causes the formation of $d$-bands that are coupled to the conduction electron bands. The bonding results from the partially filled d-bands where the average electron energy is lower than that of the free atom. There is also a repulsive interaction among the d-orbitals on neighboring ion sites causing a shift upward 
of the entire band. The banding and repulsive potentials have been calculated from tight-binding theory, (12) with parameters that may be determined entirely from a first-principles theory of the electronic structure. (13)

We will begin our discussion with the treatment of the conduction band electrons (the s-electrons) and follow the treatment given in Ref. 11 . For a transition metal with $\mathrm{N}$ ions and a total of $\mathrm{Z}$ valence electrons per ion, we consider $z_{d}$ of these to be fixed in localized nonoverlapping d-states on each ion site, and the remaining $Z_{s}=Z-Z_{d}$ electrons per ion to be in free-electron-like conduction states. The $Z_{s}$ electrons will be treated within simple metal theory using the Thomas-Fermi theory for the electron-electron screening interaction.

The total adiabatic potential is $\Phi-N I_{z}=\Omega(V)+\sum \phi(r ; V)$, as given in Eq.(II.1). The volume dependent term can be written as

$$
\Omega=Z_{s} N\left(3 / 5 \epsilon_{f}+\epsilon_{x}+\epsilon_{c}\right)+1 / 2 Z_{s} N W(q=0)+\left(N V_{a} / 2 \pi^{2}\right) \int_{0}^{\infty} F(q) q^{2} d q \text {, }
$$

where $\epsilon_{\mathrm{f}}, \epsilon_{\mathrm{X}}, \epsilon_{\mathrm{c}}$ are the free-electron gas Fermi, exchange, and correlation energies, respectively, and $v_{a}$ is the volume per ion. $W$ is the pseudopotential and $W(q=0)=-2 / 3 \epsilon_{f} . \quad F(q)$ is the energy-wavenumber characteristic ${ }^{7}$ and for Fermi-Thomas screening the integral may be calculated analytically and is given by Eq. (A16) of Ref. 11. The result is

$$
\left(N V_{a} / 2 \pi^{2}\right) \int_{0}^{\infty} F(q) q^{2} d q=-1 / 2 Z_{s}{ }^{2} e^{2} \cosh \left(\kappa r_{c}\right) e^{-\kappa r_{c}} .
$$

$\kappa$ is the Fermi-Thomas screening parameter related to the electron momentum at the Fermi surface $k_{f}$ by

$$
\kappa^{2}=4 \mathrm{e}^{2} \mathrm{k}_{\mathrm{f}}^{\mathrm{m} / \hbar \pi} \text {, }
$$

and $k_{f}{ }^{3}=3 \pi^{2} Z_{s} / V_{a}$. $\quad r_{c}$ is the empty-core pseudopotential parameter and will be determined by empirically fitting to experimental data. The first two terms of (III.I) may be written in terms of the atomic spher radius $\mathrm{r}_{0}\left(\mathrm{~V}_{\mathrm{a}}=4 \pi \mathrm{r}_{0}{ }^{3} / 3\right)$ as: $Z_{S^{N}} 3 / 5 \epsilon_{f}=2.21 \hbar^{2} / 2 \mathrm{~m} Z_{s}^{5 / 3} r_{0}{ }^{-2}, Z_{S} \epsilon_{x}=-0.916 e^{2 / 2} z^{4 / 3} r_{0}{ }^{-1}$. We will neglect the free electron correlation energy $\epsilon_{c}$.

When the perturbation expansion of the pseudopotential is approximated by the second-order term alone, we have a pair-wise effective ion potential and $\sum \phi(r ; v)=N / 2 \sum_{i}^{\prime} v_{F}\left(r_{i}\right)$, with 
$\nabla_{F}(r)=Z_{s}{ }^{2} e^{2} \cosh ^{2}\left(\kappa r_{c}\right) e^{-\kappa r} / r$

An effective d-d interaction is determined by coupling d-orbitals on different sites with the conduction electrons. This is evaluated using LCAO theory and Hartree-Fock free atom wave functions. (13) The tight binding matrix elements are represented in the theory by the quantity $r_{d}$ and there are no adjustable parameters in the theory. Values of $r_{d}$ are given in Table $I$. The potential is composed of an attractive term $V_{B}(r)$ describing the formation : $f$ bands from the atomic d-orbitals, and a repulsive term $V_{C}(r)$ describing the overlap repulsion of the d-orbitals on neighboring sites. The resulting formulae are: (11)

$$
\begin{aligned}
& V_{B}(r)=-Z_{d}\left(1-Z_{d} / 10\right)(28.1 / \pi) \hbar^{2} r_{d^{3} / m r^{5}}, \\
& V_{C}(r)=Z_{d}^{2}\left(225 / \pi^{2}\right) \hbar^{2} r_{d}^{6} / m r^{8} .
\end{aligned}
$$

$\mathrm{Z}_{\mathrm{d}}$ is the number of d-electrons. As a result of the hybridization, one finds that there are approximately $1.5 \mathrm{~s}$-electrons and $Z_{\mathrm{d}}=\mathrm{Z}-1.5 \mathrm{~d}$-electrons for all of the transition metals (see Sec. IV of Ref. 13 for further discussion); for nickel $\mathrm{Z}_{\mathrm{s}}=1.5$ and $\mathrm{z}_{\mathrm{d}}=8.5$.

TABLE I. $r_{\mathrm{d}}$ and $r_{0}$ values for the transition metals given in angstroms $(\dot{A})$

\begin{tabular}{|c|c|c|c|c|c|c|c|c|c|c|c|}
\hline & & $r_{0}$ & $r_{d}$ & & & $r_{0}$ & $\mathrm{r}_{\mathrm{d}}$ & & . & $r_{0}$ & $r_{d}$ \\
\hline \multirow[t]{9}{*}{$3 d$} & $\mathrm{Sc}$ & 1.80 & 1.163 & $4 d$ & $\mathrm{Y}$ & 1.98 & 1.602 & $5 d$ & $\mathrm{Lu}$ & 1.92 & 1.603 \\
\hline & $\mathrm{Ti}$ & 1.60 & 1.029 & & $\mathrm{Zr}$ & 1.77 & 1.415 & & $\mathrm{Hf}$ & 1.75 & 1.455 \\
\hline & $\mathrm{V}$ & 1.49 & 0.934 & & $\mathrm{Nb}$ & 1.61 & 1.328 & & $\mathrm{Ta}$ & 1.61 & 1.346 \\
\hline & $\mathrm{Cr}$ & 1.41 & $0.93 \hat{9}$ & & Mo & 1.55 & 1.231 & & $W$ & 1.56 & 1.268 \\
\hline & Mn & 1.42 & 0.799 & & Tc & 1.50 & 1.109 & & $\operatorname{Re}$ & 1.51 & 1.201 \\
\hline & $\mathrm{Fe}$ & 1.41 & 0.744 & & $\mathbf{R u}$ & 1.48 & 1.083 & & Os & 1.49 & 1.142 \\
\hline & Co & 1.39 & 0.696 & & $\mathbf{R h}$ & 1.49 & 1.020 & & $\operatorname{Ir}$ & 1.49 & 1.085 \\
\hline & $\mathrm{Ni}$ & 1.38 & 0.652 & & $\mathrm{Pd}$ & 1.51 & 1.008 & & $\mathrm{Pt}$ & 1.52 & 1.069 \\
\hline & $\mathrm{Cu}$ & 1.41 & 0.688 & & $\mathrm{Ag}$ & 1.59 & 0.889 & & $\mathrm{Au}$ & 1.58 & 1.007 \\
\hline
\end{tabular}
and taken from Ref. 13 . 


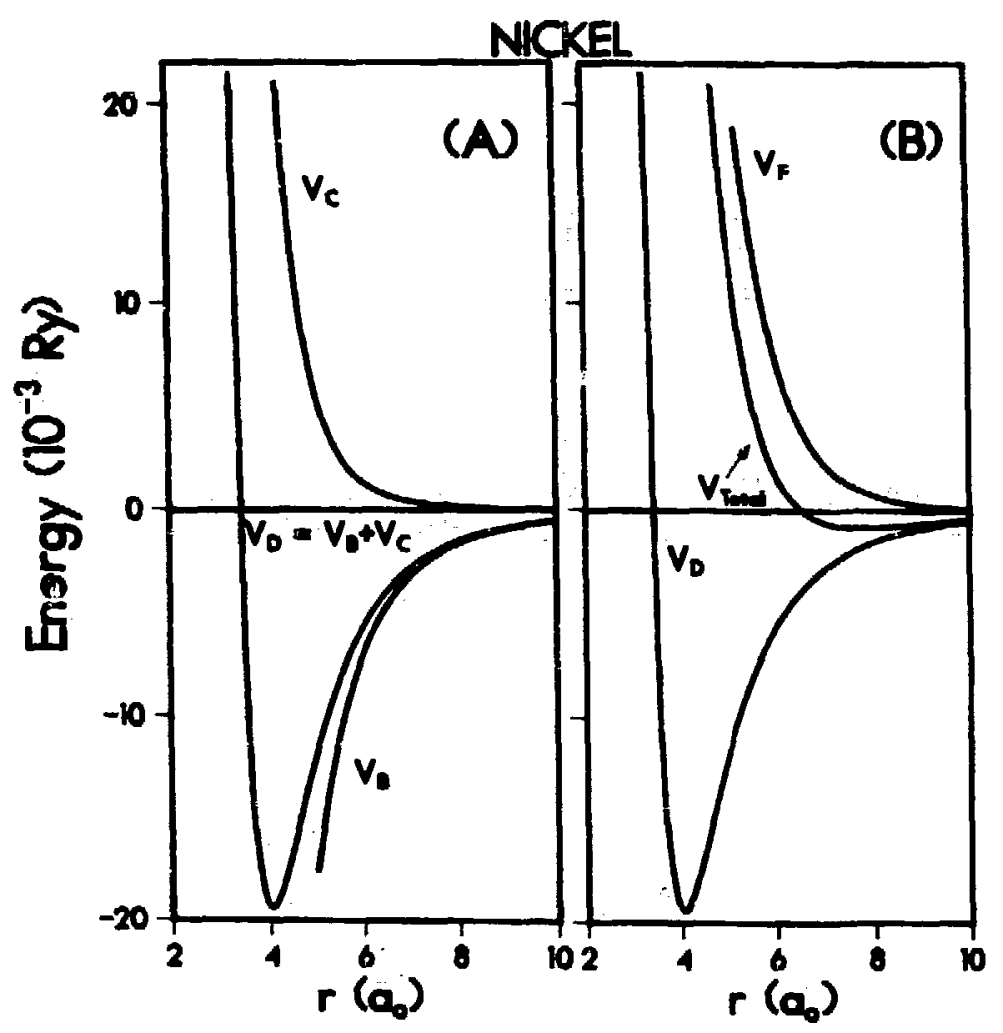

Fig.4.

EFFECTIVE ION-ION INTERACTION AND d-STATE CONTRIBUTION FOR NICKEL. (A) The effective ion-ion interaction resulting from the d-electrons, which is responsible for most of the bonding in the transition metals, is composed of an attractive term $V_{B}(r)$ describing the formation of bands from the atomic $d$-orbitals, and a repulsive term $V_{C}(r)$ describing the overlap repulsion of d-orbitals on neighboring sites. For nickel, the paremeters for Eqs. (III.3)-(III.4) are $\mathrm{z}_{\mathrm{d}}-8.5$ and $\mathrm{r}_{\mathrm{d}}-1.34 \mathrm{a}_{0}$. The total d-electron potential is $V_{d}=V_{B}+V_{C}$. (B) The conduction electrons result in an effective ion-ion interaction given by the curve labeled $V_{F}$. The parameters for Eq. (III.2) are $z_{s}=1.5$ and $r_{c}=1.4 a_{0}$ for $N i$. $v_{\text {total }}$ is the sum of the $d-$ and conduction electron terms. The total potential energy also includes a volume dependent term $\Omega(V)$ as in the simple metals.

In Fig. 4 we have plotted the effective interaction and the d-state contributions for nickel. The net d-electron potential is $V_{D}=V_{B}+V_{C}$ and the total ion-ion potential is $V_{\text {Total }}=V_{F}+V_{D}$. As is always the case for metals, the total adiabatic potential energy includes volume dependent terms in the term $\Omega(V)$, just as in the simple metals. The largest contribution to the binding energy in the transition metals comes as a result of the d-electrons.

To calculate the energy bands, we construct a Bloch sum for each type of $d$ state. For wave numbers along a cube edge in the cubic structures, there are no 


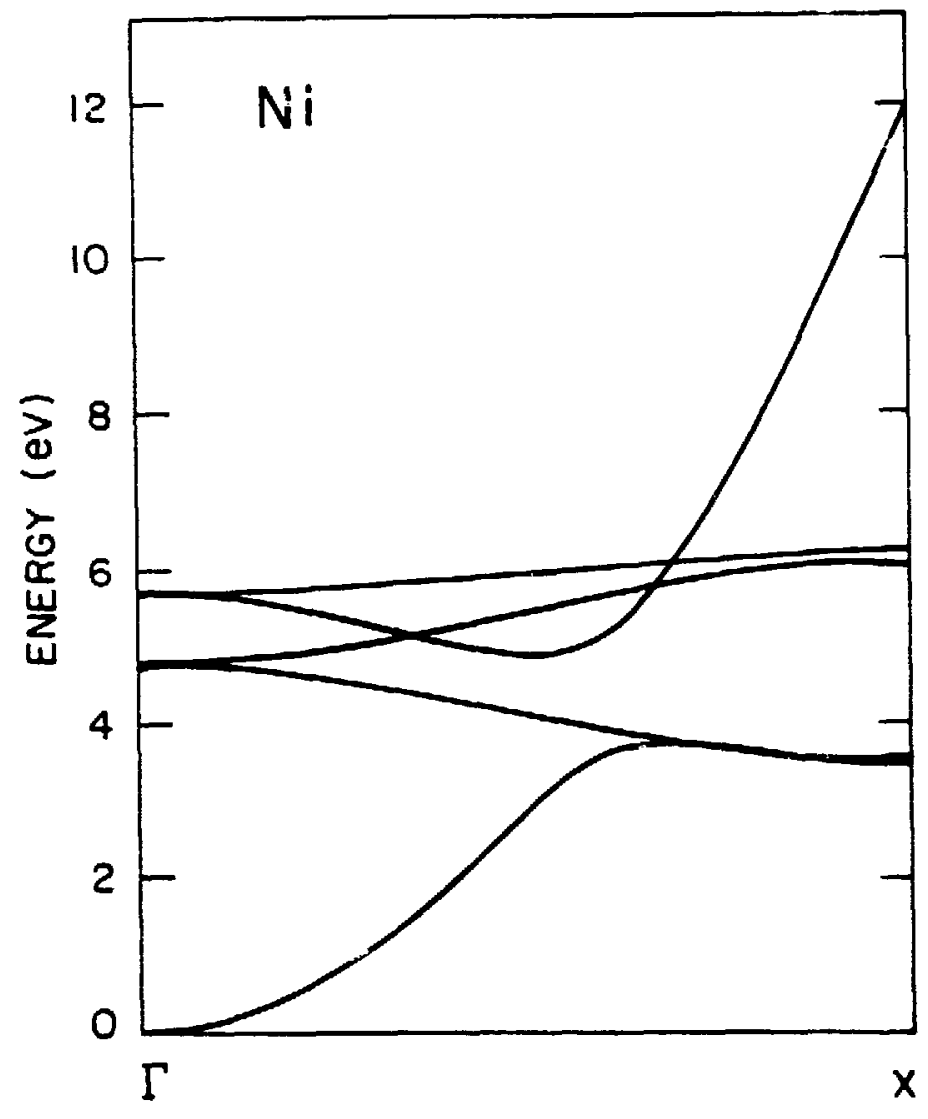

Fig. 5.

ENERGY BANDS FGR THE [001] DIRECTION IN NICKEL. The bands are plotted at the observed volume with $r_{0}=1.38 \dot{A}$. All parameters were determined from atomic wave functions and term values as given in Ref. 13.

matrix elements between Bloch sums of different d states. The basis states consist of five $d$ bands and a single plane wave. Two bands are obtained as the result of the hybridization of the plane wave and a d state, and $4 \mathrm{~d}$ bands remain uncoupled. The results for nickel are given in Fig. 5 using the atomic surface method derived in Ref. 13 and the general features of the bands are given accurately. There are no free parameters in the calculation since all quantities, such as $r_{d}$ and the position of the $d$ band center, may be determined in an approximate manner from first principles. 


\section{STATISTICAL MECHANICS OF A MD SIMULATION}

We shall pick as our starting point for discussion the end of a MD simulation where we have some set of dynamical variables A, B, ... written as functions of the sets of positions and velocities, $\{r(t)\}$ and $\{v(t)\}$, respectively. We shall only concern ourselves with systems that are in equilibrium. The initial portion of an MD run has not reached equilibrium and must be allowed to do so. The choice of the starting point for equilibrium is discussed in Ref. (14) and we will assume that this has been done properly. Briefly, equilibrium for a $\mathbb{N D}$ system is achieved when the probability distributions for all of the dynamical variables of interest are independent of time. A more useful definition is that a MD system is in equilibrium when the mean and variance of a dynamical variable are independent of time. We may also make a guess of the equilibrium starting time by a graph such as Fig. 1, but this is a subjective choice.

The total kinetic energy shown in Fig. 1 is one example of a dynamical quantity that is a function of $\{v(t)\}$. The potential energy would be an example of a dynamical quantity that is a function of only $(r(t))$. In the constant energy MD simulation that we are considering, the potential energy could be obtained by subtracting the kinetic energy from the total energy and would have exactly the same shape, but with a change in sign.

An ensemble is a very large number of systems in contact with the appropriate reservoir and with constraints for each system. Strictly speaking, in order to create a MD ensemble we would have to run a great many MD simulations with each calculation being one system. We may do this from a single MD run by invoking the quasiergodic hypothesis. (2) The quasiergodic hypothesis states that the time average of a dynamical quantity averaged over a sufficiently long period of time, such that the average is independent of the averaging time, is the same as the ensemble average.

Thermodynamic functions are defined in terms of the canonical ensemble. (15) The canonical ensemble consists of $N$ particles in a volume $V$ where $V$ is so large that boundary conditions and surface effects can be neglected. All systems in the ensemble are in contact with a reservoir at constant temperature $T$. For the MD ensemble we have constant $N$ and $V$, but neither of these quantities will be large enough to neglect the surfaces or boundary conditions. The reservoir for 
each MD system is an angular momentum reservoir. (1) The path to equilibrium thermodynamics from MD consists of defining thermodynamic functions as averages over a canonical ensemble, relating the canonical ensemble averages to MD ensemble averages, and creating the MD ensemble by taking the time average of a single MD system.

\section{A. Thermodynamic Functions in the Classical Canonical Ensemble}

We will follow the approach of Wallace(2) for the discussion of the canonical ensemble and the definition of thermodynamic functions. For the canonical ensemble, we have constant $N, V$, and a reservoir that keeps each system at constant $T$. The canonical weight function is

$\mathrm{W}(\beta) \propto \mathrm{e}^{-\beta \mathrm{H}}$,

where $\beta=1 / \mathrm{kT}$ and $\mathrm{k}$ is Boltzmann's constant. $\mathrm{H}$ is the system Hamiltonian such that $H=K . E .+\Phi$ and K.E. is the total system kinetic energy. The classical canonical partition function is

$$
\mathrm{Z}(\beta)=1 / \mathrm{N} ! \mathrm{h}^{3 \mathrm{~N}} \int \cdot \cdot \int \mathrm{e}^{-\beta \mathrm{H}} \mathrm{\Pi}_{\mathrm{k}} \mathrm{dx}_{\mathrm{k}} \mathrm{d}_{\mathrm{k}} .
$$

$\mathrm{P}_{\mathrm{k}}$ is the momentum coordinate of particle $\mathrm{k}$. For a fluid, each positional coordinate $x_{k}$ is integrated over the entire volume. In a crystal each particle coordinate may be restricted to the volume surrounding a lattice site, and then $Z$ must be multiplied by $N$ ! to count the particle permutations. The partition function may be written in the form

$$
\mathrm{Z}(\beta)=\left(\mathrm{V}^{\mathrm{N}} / \mathrm{N} ! \Lambda^{3 \mathrm{~N}}\right) \mathrm{Q}(\beta),
$$

where $\Lambda^{2}=\left(2 \pi \hbar^{2} / \mathrm{mkT}\right)^{1 / 2}$. is the thermal de Broglie wavelength of the particles. The configuration integral $Q(\beta)$ is

$$
\mathrm{Q}(\dot{\beta})=\underset{\cdots}{1} / \mathrm{V}^{\mathrm{N}} \int \cdots \int \mathrm{e}^{-\beta \Phi} \Pi_{\mathrm{k}} \mathrm{dx} \mathrm{x}_{\mathrm{k}} .
$$

Thermodyamic quantities can be connected to the statistical mechanics of the ensemble through a single equation involving the Helmholtz free energy $F$ :

$$
\mathrm{F}=-\mathrm{kT} \ln \mathrm{Z}(\beta) \text {, }
$$

and all remaining thermodynamic functions are obtained from $F$. The entropy $S$ is $S=-[\partial F / \partial T] V$,

the internal energy $U$ is

$\mathrm{U}=\mathrm{F}-\mathrm{TS}$,

and the pressure $\mathrm{P}$ is

$\mathrm{P}=-[\partial \mathrm{F} / \partial \mathrm{V}]_{\mathrm{T}}$. 
The functions $S, U$, and $P$ can $a 11$ be shown to be given by ensemble averages. It is important to note that $\beta$ is a parameter that identifies a particular canonical ensemble (along with $N, V$ ). If we note the canonical ensemble average of a dynamical quantity $A$ by $\langle A \mid \beta\rangle$, and define $b=-\beta \vec{u}$ where $\vec{u}$ is the mean center-of-mass velocity, then Ref. (1) gives the following results:

$\langle\mathrm{H} \mid \beta\rangle=\mathrm{U}+\mathrm{Nmb}^{2} / 2 \beta^{2}$,

$\langle\mathrm{K} . \mathrm{E} . \mid \beta\rangle=3 / 2 \mathrm{kT}+\mathrm{Nmu}^{2} / 2$.

For a metal with a Hamiltonian $H=$ K.E. $+\Phi$ we rewrite the adiabatic potential assuming pair interactions only. Then,

$$
\Phi=N I_{z}+\Omega(V)+1 / 2 \sum_{i, j} \phi\left(r_{i j} ; V\right) \quad(i \neq j) .
$$

The ensemble average of the pressure is given by

$$
P=-d \Omega / d V+N k T / V-\langle Y \mid \beta\rangle / V \text {, }
$$

where $\mathrm{Y}$ is the generalized virial function given by

$$
Y=1 / 2 \sum_{i, j}\left(V \partial \phi / \partial V+1 / 3 \quad r_{i j} \partial \phi / \partial r_{i j}\right) \text {. }
$$

For the dynamical variable $A$, the fluctuation of $A$ is given by $\delta A=A-\langle A\rangle$ where the average is over a particular ensemble. Canonical ensemble averages of the fluctuations of the potential and kinetic energies are

$\langle\delta \Phi \delta \mathrm{K} . \mathrm{E} . \mid \beta\rangle=0$,

$<(\delta \mathrm{K} . \mathrm{E} .)^{2}|\beta\rangle=3 / 2 \mathrm{~N}(\mathrm{kT})^{2}+\mathrm{Nm}^{2} \overrightarrow{\mathrm{b}}^{2} / \beta^{3}$,

$\left\langle(\delta \Phi)^{2} \mid \beta\right\rangle=\mathrm{N}(\mathrm{kT})^{2}(\mathrm{c}-3 / 2)$.

$c=1 / \mathrm{Nk}[\partial U / \partial \mathrm{T}] \mathrm{V}$ is the heat capacity per particle at constant volume.

\section{B. Ensemble Corrections}

We are able to calculate dynamical quantities using ML and form a MD ensemble from the time averages using the quasiergodic hypothesis. We have defined the thermodynamic functions in terms of a different ensemble, namely, the canonical ensemble. These ensembles are different and averages of some dynamical quantity for the two ensembles will be different. Since we want to obtain highly accurate thermodynamic functions (in the canonical ensemble), but we have dynamical quantities calculated in the MD ensemble, we will need to make corrections between the two. These corrections are given in detail in Ref. (1). Here we will merely state the results.

For any dynamical quantity involving only averages of the ensemble, then

$$
\langle A\rangle_{M D}=\langle A \mid \beta\rangle+O(1 / N) \text {. }
$$

$O(1 / N)$ refers to corrections of order $1 / N$ where for a $N=100$ MD calculation, the 
correction would be $18, \mathrm{~N}=1000,0.1 \%$, etc. The kinetic energy averaged over the canonical ensemble is $3 / 2 \mathrm{NkT}$ (for a mean center-of-mass velocity equal to zero). Then,

$$
\begin{aligned}
& <\mathrm{K} . \mathrm{E} .>_{\mathrm{MD}}=3 / 2 \mathrm{NKT}+0(1 / \mathrm{N}), \\
& \mathrm{U}_{\mathrm{MD}}=\left\langle\mathrm{K} . \mathrm{E} .>_{\mathrm{MD}}+\langle\Phi\rangle_{\mathrm{MD}}+0(1 / \mathrm{N}),\right. \\
& \mathrm{P}_{\mathrm{MD}}=-\mathrm{d} \Omega / \mathrm{dV}+<2 / 3 \mathrm{~V} \text { K.E. }-\mathrm{Y}_{\mathrm{MD}}+0(1 / \mathrm{N}) .
\end{aligned}
$$

The formula relating the pressure in the $M D$ and canonical ensembles is

$$
\mathrm{P}_{\mathrm{MD}}=\mathrm{P}-\mathrm{kT} / \mathrm{V}(1-3 / 2 \gamma+\mathrm{T} / 2 \quad \partial \gamma / \partial \mathrm{T}) \text {. }
$$

$y=\mathrm{V}[\partial \mathrm{P} / \partial U]_{\mathrm{V}}$ is the Gruneisen parameter. The last term in this equation is of relative order $1 / \mathrm{N}$ with respect to $\mathrm{P}$.

For fluctuations of dynamical quantities, the ensemble corrections are of $O(1)$ and must be included. For instance,

$$
\begin{aligned}
& \left.<(\delta \mathrm{K} . \mathrm{E} .)^{2}\right\rangle_{\mathrm{MD}}=3 \mathrm{~N} / 2(\mathrm{kT})^{2}(1-3 / 2 \mathrm{c}), \\
& \langle\delta \mathrm{Y} \delta \Phi\rangle_{\mathrm{MD}}=3 \mathrm{~N} / 2(\mathrm{kT})^{2}(1 / \mathrm{c}-\gamma) .
\end{aligned}
$$

The calculation of the elastic constants from MD requires the evaluation of fluctuations such as in these equations. The ensemble corrections have been worked out in Ref. (3).

\section{CALCULATION OF THERMODYNAMIC QUANTITIES}

MD simulations can give "exact"(16) solutions to the problem of solving for the vibrational motion of a system of point particles interacting with some potential that depends upon the relative positions of all the particles. In the last section, the construction of the MD statistical ensemble was discussed as well as the definition of thermodynamic functions in the classical canonical ensemble. Relationships between the MD and canonical ensemble were given along with formulas for the calculation of ensemble corrections. We are now ready to proceed with a more detailed analysis of the MD results.

The strength of MD simulations lies in their ability to produce "exact" answers that can augment or extend other theoretical approaches. This situation is well exemplified for the study of thermodynamics in solids where the theory of lattice dynamics in the quasiharmonic approximation gives amazingly accurate results at low to moderate temperatures, generally up to the Debye temperature. In fact, we shall see that since MD is solving classical equations of motion, it will give wrong answers below the Debye temperature. Above the Debye 
temperature, the vibrational behavior of the ions can be adequately described as classical point particles and then MD can give extremely reliable and useful information. In the solid phase, we will use the theory of lattice dynamics to analyze the MD results. The necessary information needed from lattice dynamics will be presented in Section VI.

\section{A. The Internal Energy and Pressure}

From an equilibrium MD simulation at constant volume, we are able to calculate the total energy and pressure by making time averages (indicated by <MD) of the kinetic energy, potential energy, and the generalized virial. The total internal energy is given by

$$
\mathrm{U}_{\mathrm{MD}}=\langle\mathrm{K} . \mathrm{E} .\rangle_{\mathrm{MD}}+\langle\Phi\rangle_{\mathrm{MD}} \text {, }
$$

where from Eq.(1), we may write out the last term for $\phi$ as a two-body central potential $\left(r_{i j}=r_{i}-r_{j}\right)$ :

$$
\left.\langle\Phi\rangle_{M D}=N I_{z}+\Omega(V)+1 / 2 \sum_{i, j} \phi\left(r_{i j} ; V\right)\right\rangle_{M D},(i \neq j) \text {. }
$$

The total pressure is

$$
\mathrm{P}_{\mathrm{MD}}=-\mathrm{d} \Omega / \mathrm{dV}+2 / 3 \mathrm{~V}<\mathrm{K} . \mathrm{E} .>_{\mathrm{MD}}-\langle\mathrm{Y}\rangle_{\mathrm{MD}} / \mathrm{V} \text {. }
$$

$\mathrm{Y}$ is the generalized virial for a volume dependent potential and is

$$
Y=1 / 2 \sum_{i, j}\left(V \partial \phi / \partial V+1 / 3 r_{i j} \partial \phi / \partial r_{i j}\right) \text {. }
$$

From Eq. (19) we can calculate the temperature and with $M$ the particle mass we have

$$
\left.\mathrm{kT}=2 / 3 \mathrm{~N}<\mathrm{K} . \mathrm{E} \cdot>_{\mathrm{MD}}=\mathrm{M} / 3 \mathrm{~N}<\sum_{\mathrm{i}} \mathrm{v}_{\mathrm{i}}{ }^{2}\right\rangle_{\mathrm{MD}}
$$

In Fig. 6, the results for the total internal energy and the total pressure at several different values of the initial energy are given as the solid points for three different volumes. The temperature was determined at the end of each run using the preceding equation. The break in each curve separates runs whose final equilibrium state was in the solid phase from those in the fluid phase. All calculations were started at time zero with all particles on bcc lattice sites and a random distribution of velocities. The time evolution of the kinetic energy for two such calculations is shown in Fig. 1. 

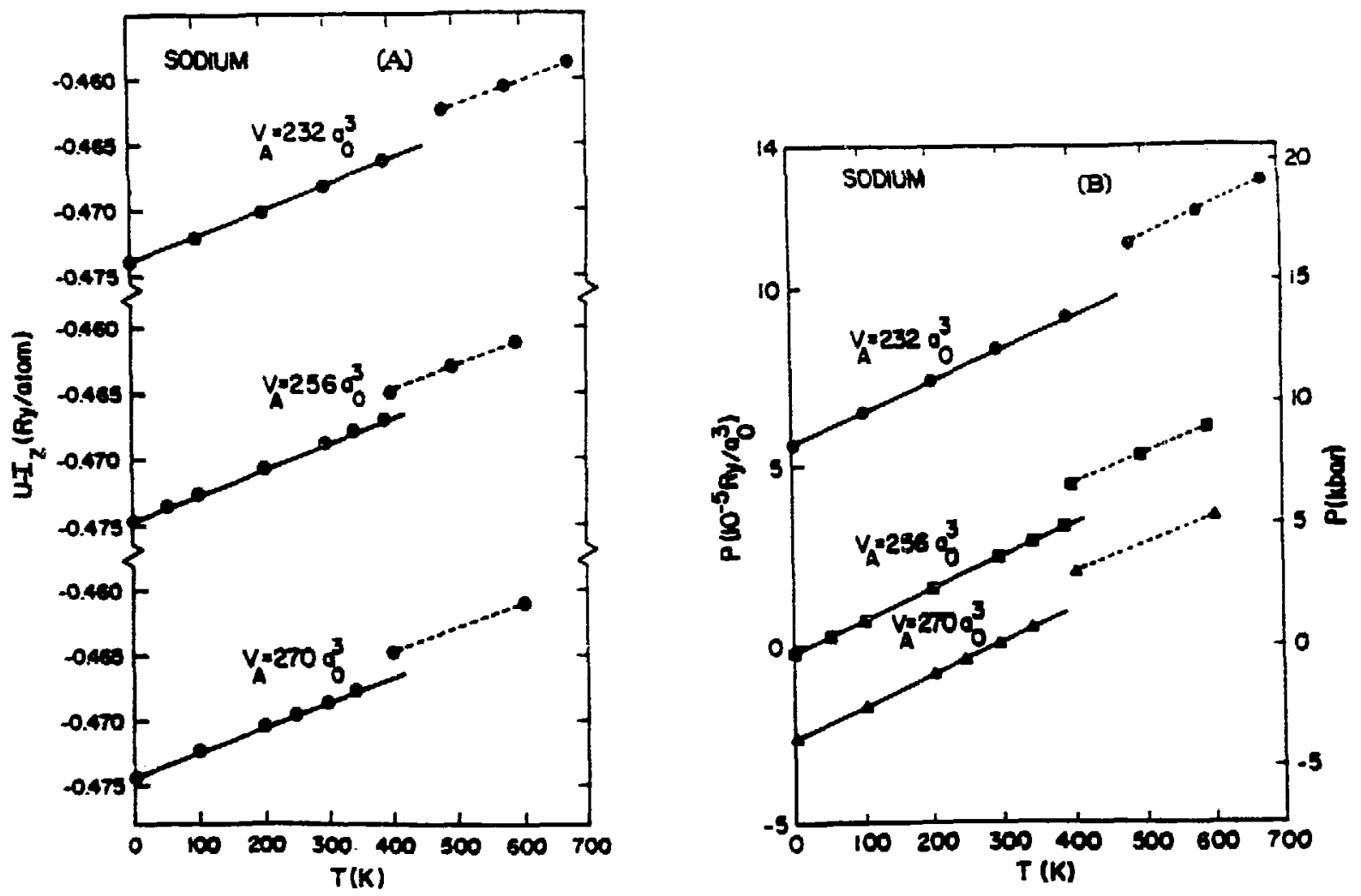

Fig. 6.

MD RESULTS FOR THE TOTAL INTERNAL ENERGY AND THE TOTAL PRESSURE. The points are the results of $M D$ calculations at three different volumes. (A) The total interrial energy per atom $U$ minus the ionization energy $I_{z}$ vs temperature from the $\mathrm{MD}$ calculations. The solid lines are the quasiharmonic part of $\mathrm{U}-\Phi_{0}+3 \mathrm{HkT}$ $+U_{A}$; i.e., they express the quantity $\Phi_{0}-\mathrm{NI}_{Z}+3 \mathrm{NkT}$. $\Phi_{0}$ is $\Phi$ evaluated at $\mathrm{T}-0$. (B) The total pressure $P$ vs temperature from the MD calculations. The solid lines are the quastharmonic part of $P_{-}-d \Phi_{0} / d V-3 N k T d l n \theta / d V+P_{A}$. The dashed Iines through the fluid phase points are not theoretically derived. The right hand scale is in units of kilobars; $T$ is in units of Kelvins.

\section{B. Free Energy}

In Section IV the Helmholtz free energy $F$ was used to define all the thermodynamic functions. We shall now write $F$ in terms of a harmonic part $F_{H}$ and an anharmonic part $F_{A}$. $F_{H}$ can be calculated in the high temperature limit of quasiharmonic lattice dynamics theory as given in Section $\mathrm{vI}$. The result is

$$
\begin{aligned}
& F=F_{H}+F_{A}, \\
& F=\Phi_{0}-3 N k T \ln (T / \theta)+F_{A},
\end{aligned}
$$

where 


$$
\ln (k \theta)=\langle\ln \hbar \omega\rangle_{\mathrm{BZ}}
$$

$<->_{\mathrm{BZ}}$ is an average of the Brillouin zone of the phonon frequencies calculated for the central potential $\phi(r ; V)$. We may calculate the other thermodynamic functions from $F$ and for the internal energy $U$, we have

$$
\begin{aligned}
& \mathrm{U}=\mathrm{F}-\mathrm{TS}=\mathrm{F}-\mathrm{T}[\partial \mathrm{F} / \partial \mathrm{T}]_{\mathrm{V}}, \\
& \mathrm{U}=\Phi_{0}+3 \mathrm{NkT}+\mathrm{U}_{\mathrm{A}},
\end{aligned}
$$

where the harmonic part is $\mathrm{U}_{\mathrm{H}}=\Phi_{0}+3 \mathrm{NkT}$ and the anharmonic part

$$
\mathrm{U}_{\mathrm{A}}=\mathrm{F}_{\mathrm{A}}-\mathrm{T}\left[\partial \mathrm{F}_{\mathrm{A}} / \partial \mathrm{T}\right]_{\mathrm{V}}
$$

The pressure may be similarly calculated from $F$ and written as harmonic and anharmonic contributions.

$$
\begin{aligned}
& \mathrm{P}=-[\partial \mathrm{F} / \partial \mathrm{V}]_{\mathrm{T}}-\left[\partial \mathrm{F}_{\mathrm{H}} / \partial \mathrm{V}\right]_{\mathrm{T}}-\left[\partial \mathrm{F}_{\mathrm{A}} / \partial \mathrm{V}\right]_{\mathrm{T}}=\mathrm{P}_{\mathrm{H}}+\mathrm{P}_{\mathrm{A}} . \\
& \mathrm{P}_{\mathrm{H}}=-\mathrm{d} \Phi_{0} / \mathrm{dV}-3 \mathrm{Nkdln} \theta / \mathrm{dV}, \\
& \mathrm{P}_{\mathrm{A}}=-\left[\partial \mathrm{F}_{\mathrm{A}} / \partial \mathrm{V}\right]_{\mathrm{T}} .
\end{aligned}
$$

The equations for the harmonic and anharmonic portions of the entropy are

$$
\mathrm{S}=-[\partial \mathrm{F} / \partial \mathrm{T}]_{\mathrm{V}}=\mathrm{S}_{\mathrm{H}}+\mathrm{S}_{\mathrm{A}}
$$

$\mathrm{S}_{\mathrm{H}}=3 \mathrm{Nk}+3 \mathrm{Nk} \ln (\mathrm{T} / \theta) ; \mathrm{S}_{\mathrm{A}}=-\left[\partial \mathrm{F}_{\mathrm{A}} / \partial \mathrm{T}\right]_{\mathrm{T}}$.

At high temperatures, there are also contributions from the thermal excitation of the electrons from the ground state. These are particularly important in the fluid and we give the appropriate formulas here.(17) The electronic internal energy for the conduction electron gas is given by

$$
\mathrm{U}_{\mathrm{e}}=1 / 2 \Gamma \mathrm{T}^{2} \text {, }
$$

and the pressure is given by

$$
\mathrm{P}_{\mathrm{e}}=2 / 3 \mathrm{U}_{\mathrm{e}}=1 / 3 \Gamma \mathrm{T}^{2}
$$

$\Gamma$ was evaluated in the free-electron approximation from the expression

$$
\Gamma-\mathrm{N} \pi^{2} \mathrm{k}^{2} \mathrm{mZ} / \hbar^{2} \mathrm{k}_{\mathrm{f}}^{2} \text {, }
$$

where $m$ is the electron mass and $k_{f}$ is the free-electron Fermi wave number. Because of the fairly strong temperature dependence of the electronic terms, they cannot be neglected in the fluid phase and are necessary for good agreement with experiment. In metallic fluid sodium, $\mathrm{P}_{\mathrm{e}}$ becomes abolit 38 of the ionic contribution to the pressure at $\mathrm{T}=1800 \mathrm{~K}$.

\section{Fitting the Molecular Dynamics Data}

The solids lines in Fig. 6 are from $U_{H}$ and $P_{H}$ and lie very close to the $M D$ points. We are interested in the deviations of the MD points from the straight line. The MD results must be accurate enough to determine such deviations. 
Otherwise, we can obtain all thermodynamic quantities from quasiharmonic lattice dynamics theory and do not need to do a MD simulation at all. More importantly, Eqs.(V.1)-(V.4) suggest a simple technique to extract the maximuin information from MD and extremely accurate thermodynamics. Since we believe that the total pressure and energy are correct from the MD ralculations (denoted as $P_{M D}$ and $\left.U_{M D}\right)$, then the anharmonic quantities ars given by

$$
\begin{aligned}
& \mathrm{U}_{\mathrm{A}}=\mathrm{U}_{\mathrm{MD}}-\mathrm{U}_{\mathrm{H}}, \\
& \mathrm{P}_{\mathrm{A}}=\mathrm{P}_{\mathrm{MD}}-\mathrm{P}_{\mathrm{H}} .
\end{aligned}
$$

This has bein done for bec sodium and the results are given in Fig. 7 .

The solid curves are the results of numerical fits of $U_{A}$ and $P_{A}$ using all the MD results. The functional form of this fit is determined by studying the behavior of the high temperature expansion of the anharmonic free energy which suggests that $F_{A}$ should be a power series expansion of leading order $T^{2}$. Accordingly, we let

$$
F_{A}=A_{2}(V) T^{2}+A_{3}(V) T^{3}+A_{4}(V) T^{4} \text {, }
$$

where each of the coefficients have explicit volume dependence, but no further temperature dependence. The $A_{i}(V)$ may be expanded as a power series in the volume $\mathrm{V}$ which we took to be

$$
-A_{i}(V)=d_{i 0}+d_{i 1} V+d_{i 2} V^{2} \text {, }
$$

for $i=2,3,4$. Then the anharmonic internal energy is

$$
\mathrm{U}_{\mathrm{A}}=-\mathrm{A}_{2} \mathrm{~T}^{2}-2 \mathrm{~A}_{3} \mathrm{~T}^{3}-3 \mathrm{~A}_{4} \mathrm{~T}^{4}
$$

The anharmonic pressure ist

$$
P_{A}=-\left(d A_{2} / d V\right) T^{2}-\left(d A_{3} / d V\right) T^{3}-\left(d A_{4} / d V\right) T^{4},
$$

and the anharmonic entropy, is

$$
S_{A}=-2 A_{2} T-3 A_{3} T^{2}-4 A_{4} T^{3} \text {. }
$$

The alternative to the analysis given here is to take the.MD points directly and numerically fit them to some functional representation of the Helmholtz free energy that allows one to easily take derivatives with respect to $V$ and $T$. Although certainly possible and even required for the fluid phase, one loses a great deal of information and understanding of the relative contributions in the vibrational behavior for the thermodynamic functions. 

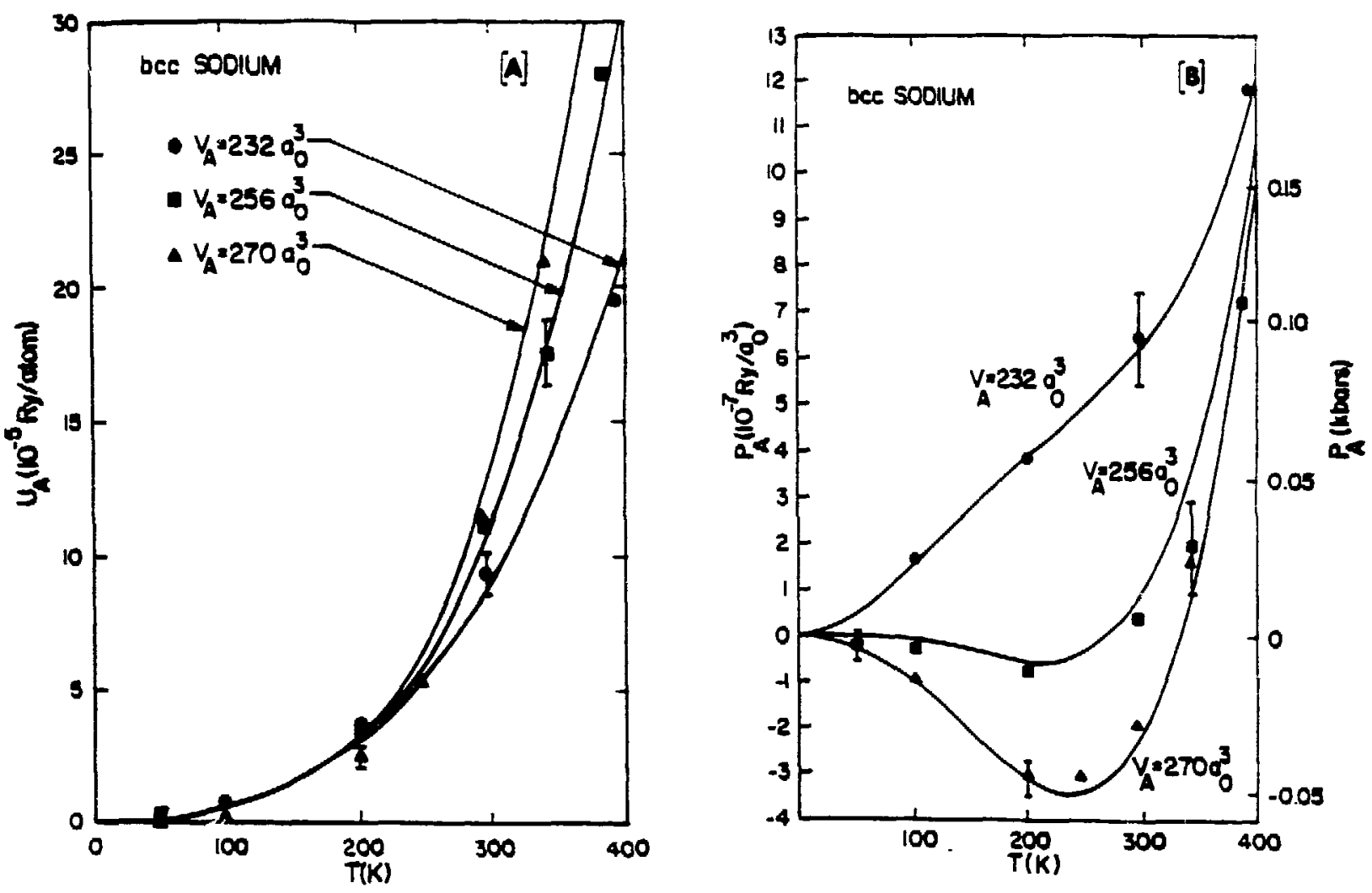

Fig. 7.

ANHARMONIC INTERNAL ENERGY AND PRESSURE VS TEMPERATURE. The anharmonic contributions to the (A) internal energy $U_{A}$ and $(B)$ the pressure $P_{A}$ at three different volumes. The solid curves are from the fits to al' the MD results for the internal energy and pressure by fitting the coefficients in Eq. (V.7).

\section{Thermodynamic Functions}

We can now use the formulas of (V.5)-(V.11) to evaluate any thermodynamic function of interest. Refs. (9), (10), (18) contain the results for the evaluation and comparison with experiment of the thermodynamic functions for bcc sodium. Here we will give the results for the isothermal bulk modulus $\mathrm{B}_{\mathrm{T}}$, heat capacity at constant volume $C_{V}$ and pressure $C_{P}$, and the thermodynamic Gruneisen parameter $\gamma$. The isothermal bulk modulus is given by

$$
\mathrm{B}_{\mathrm{T}}=-\mathrm{V}[\partial \mathrm{P} / \partial \mathrm{V}]_{\mathrm{T}}=-\mathrm{V}\left(-\mathrm{d}^{2} \Phi_{\mathrm{O}} / \mathrm{dV}^{2}-3 \mathrm{NkTd^{2 }} \ln \theta / d \mathrm{~V}^{2}+\left[\partial \mathrm{P}_{\mathrm{A}} / \partial \mathrm{V}\right]_{\mathrm{T}}\right],
$$

where the anharmonic contribution is

$$
-V\left[\partial \mathrm{P}_{\mathrm{A}} / \partial \mathrm{V}\right]_{\mathrm{T}}=-\left(\mathrm{d}^{2} \mathrm{~A}_{2} / d V^{2}\right) \mathrm{T}^{2}-\left(\mathrm{d}^{2} \mathrm{~A}_{3} / d V^{2}\right) \mathrm{T}^{3}-\left(\mathrm{d}^{2} \mathrm{~A}_{4} / d V^{2}\right) \mathrm{T}^{4}
$$


The anharmonic contribution is not entirely negligible, but is only a few percent from $\mathrm{T}=200 \mathrm{~K}$ to melting for sodium.

The constant volume heat capacity is

$$
\begin{aligned}
C_{\mathrm{V}}= & {[\partial \mathrm{U} / \partial \mathrm{T}]_{\mathrm{V}} 3 \mathrm{Mk}+\left[\partial \mathrm{U}_{\mathrm{A}} / \partial \mathrm{T}\right]_{\mathrm{T}}, } \\
& =3 \mathrm{Nk}-2 \mathrm{~A}_{2} \mathrm{~T}-6 \mathrm{~A}_{3} \mathrm{~T}^{2}-12 \mathrm{~A}_{4} \mathrm{~T}^{3},
\end{aligned}
$$

The heat capacity at constant pressure is

$$
\mathrm{C}_{\mathrm{P}}=\mathrm{T}[\partial \mathrm{S} / \partial \mathrm{T}]_{\mathrm{P}}
$$

When $P=0$, then $d U=T d S$ and $C_{F}(P=0)=[\partial U / \partial T]_{P=0}$. This will not produce the same formula as Eq. (V.12) since the $P=0$ constraint is not equivalent to the $V=$ constant constraint; the correct treatment of the temperature dependence of the volume is required.

The thermodynamic Groneisen parameter is

$$
\begin{aligned}
& \gamma=[\partial \mathrm{P} / \partial \mathrm{U}]_{\mathrm{V}}=[\partial \mathrm{P} / \partial \mathrm{T}]_{\mathrm{V}} /[\partial \mathrm{U} / \partial \mathrm{T}]_{\mathrm{V}}, \\
& {[\partial \mathrm{P} / \partial \mathrm{T}]_{\mathrm{V}}-3 \mathrm{Nk} \mathrm{dln} \theta / \mathrm{dV}+\left[\partial \mathrm{P}_{\mathrm{A}} / \partial \mathrm{T}\right]_{\mathrm{V}},} \\
& {\left[\partial \mathrm{P}_{\mathrm{A}} / \partial \mathrm{T}\right]_{\mathrm{V}}=-2\left(\mathrm{dA}_{2} / \mathrm{dV}\right) \mathrm{T}-3\left(\mathrm{dA}_{3} / \mathrm{dV}\right) \mathrm{T}^{2}-4\left(\mathrm{dA}_{4} / \mathrm{dV}\right) \mathrm{T}^{3},}
\end{aligned}
$$

and $[\partial U / \partial T]_{V}-C_{V}$ from above.

The heat capacity at constant pressure is plotted in Fig. 8 for solid sodium from $T=0 \mathrm{~K}$ to the melting temperature $\mathrm{T}_{\mathrm{M}}$. This figure illustrates the success of MD above the Debye temperature and its failure below where a quantum mechanical treatment is required. The curve in Fig. 8 labeled "classical Theory" is the MD based result; the curve labeled "Quantum Theory" comes from quasiharmonic lattice dynamics. The experimenta1 data is plotted as measured and with a correction that is appropriate for the situation where there are no vacancies. The MD simulations do not contain vacancies near $T_{M}$. At low temperatures, MD will always give the classical value of $3 \mathrm{Nk}$. In this region one must use lattice dynamics theory. Above the Debye temperature, classical theory becomes a good approximation and the MD calculations are meaningful. MD is able to correctly include the many-body anharmonicity with considerable accuracy. Even though the anharmonic contributions are small as may be seen in Fig. 7, they are primarily responsible for the excellent agreement with experiment above the Debye temperature. One should note that the same potential is used for the quantum and MD calculations.

The thermodynamic Graneisen parameter $\gamma$ as a function of temperature is plotted in Fig. 9 using Eqs.(V.13)-(V.15). $\gamma$ may be thought of as being a highly anharmonicity dependent quantity since it involves high order derivatives of 


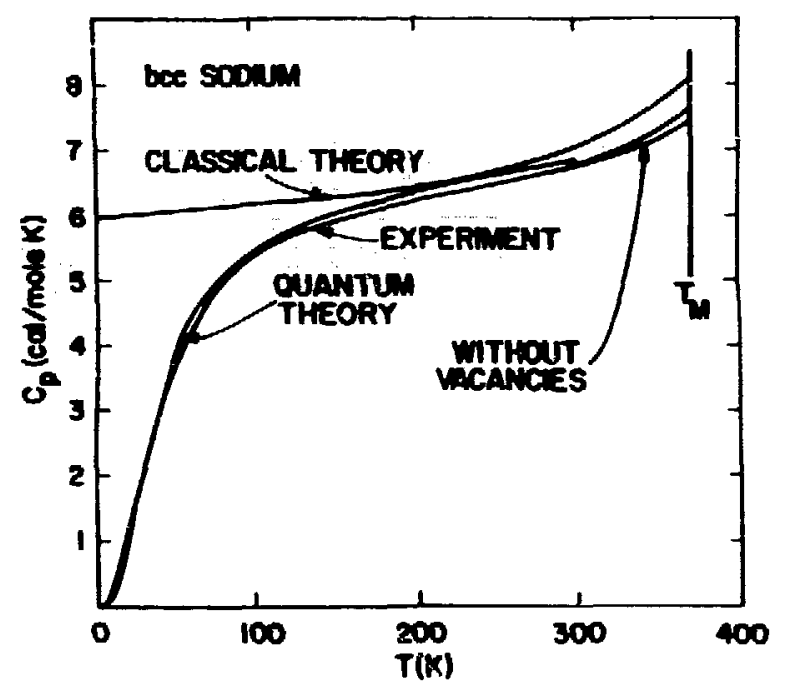

Fig. 8.

HEAT CAPACITY AT CONSTANT PRESSURE $C_{P}$ IN SOLID SODIUM. The heat capacity vs temperature is plotted from classical theory using MD, quantum theory using quasiharmonic lattice dynamics, and compared with experiment. The experimental results have been corrected to the case without vacancies as indicated near $T_{M}$.

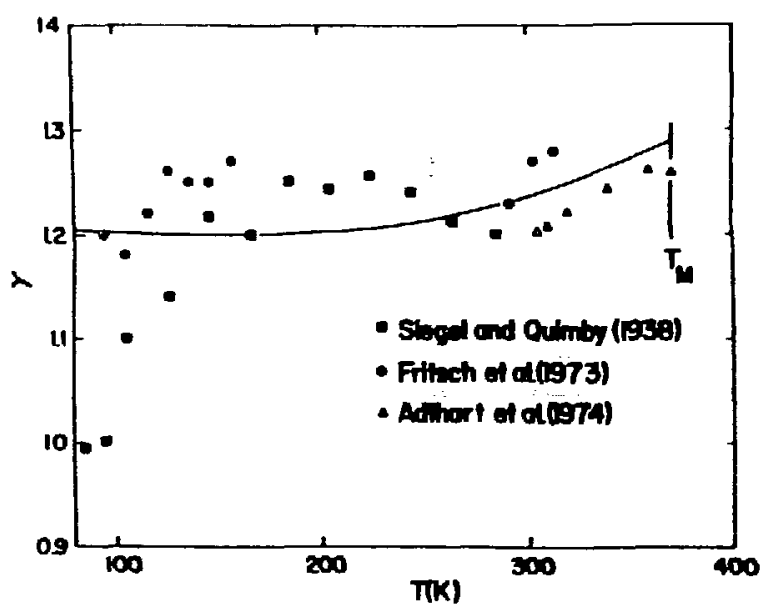

Fig. 9.

GRUNEISEN PARAMETER $\boldsymbol{\gamma}$ VS TEMPERATURE IN THE SOLID. The points are determined from the experimental values of the thermal expansion $\beta$, isothermal bulk modulus $\mathrm{B}_{\mathrm{T}}$, and heat capacity at constant volume $C_{V}$ using $\gamma_{\text {exp }}=V \beta \mathrm{B}_{\mathrm{T}} / C_{V}$. The solid line was calculated from Eqs. (V.13)-(V.15).

differentials of the pressure and the internal energy. Near $T_{M}$, the slopes of $\mathrm{U}_{\mathrm{A}}$ and $\mathrm{P}_{\mathrm{A}}$ increase rapidly. The agreemeit with experiment, both the qualitative shape and the numerical values, is excellent. The points are determined from the experimental values of the thermal expansion $\beta$, isothermal bulk modulus $\mathrm{B}_{\mathrm{T}}$, and heat capacity at constant volume $C_{V}$ using the expression

$$
\gamma_{\exp }=\mathrm{V} \beta B_{\mathrm{T}} / C_{\mathrm{V}}
$$

\section{E. The Metallic Fluid Phase}

If one increases the energy in a MD simulation sufficiently high, then the final equilibrium state for the system will be in the fluid phase as may be seen on the right hand sides of Fig. 6 (A) and (B). MD will give the total internal energy and pressure as a function of temperature at constant volume. This has been done for a large number of volumes and temperatures in the fluid and the results are given in Ref. (17). The electronic contributions to the internal 
energy and pressure, $\mathrm{U}_{\mathrm{e}}$ and $\mathrm{P}_{\mathrm{e}}$ as given in Section $\mathrm{V} . \mathrm{B}$ need to be included.

Unfortunately, we do not have a theory comparable to quasiharmonic lattice to use as a guide in the fluid phase. In the present case, $U$ and $P$ were fitted to cubic polynomials as a function of temperature at each volume. From these fits $C_{V}, \gamma, B_{T}$, and

$$
\mathrm{C}_{\mathrm{P}}=\mathrm{C}_{\mathrm{V}}+\mathrm{T}^{2} \mathrm{C}_{\mathrm{V}}^{2} / \mathrm{VB}_{\mathrm{T}}
$$

were evaluated. These quantities may be evaluated without constructing the Helmhoitz free energy. Below we shall discuss how $F$ and the fluid phase entropy constant are obtained from the high-temperature cluster expansion of the pressure and energy.

For the fluid phase, we only present the results for $C_{V}, C_{P}$, and $\gamma$ and the comparison with experiment in Figs. 10 and 11. Agreement with experiment is quite good. If one were to plot $C_{P}$ for the solid and fluid phase on the same graph, then the characteristic lambda shape would be seen at $T_{M}$. The slope of the heat capacity changes sign in passing from the solid to the fluid. The agreement between the MD result and experiment for the Gruneisen parameter is not as good as for the solid. However, the change in slope of $y$ upon melting is correctly given in the fluid.

The potential used in the fluid calculations was the same as for all of the solid calculations. It is quite remarkable that a single potential is capable of giving reliable results from $T=0 \mathrm{~K}$ to about three times $T_{M}$. At some point, the approximations of the pseudopotential will begin to break down, but an analysis of detailed electronic band structure calculations shows that the approximations should hold to above $2000 \mathrm{~K}$. In comparing the $\mathrm{P}=0$ vs $\mathrm{T}$ curve with experiment, at $\mathrm{T}=2100 \mathrm{~K}$ the entire discrepancy was equivalent to a $2.1 \mathrm{kbar}$ difference in the total pressure. Thus we conclude that the pseudopotential method is valid for volumes investigated.

\section{F. Entropy Constant of a Metallic Fluid}

In the theory of classical statistical mechanics, the value of the entropy is not unique, but only defined to within an arbitrary constant. In the solid, we start with a quantum mechanical theory and make a high temperature expansion of $F$ (see the high temperature expansion of $F_{H}$ in Section VI). This expansion, which contains the entropy constant, is the basis of the connection to the classical equations of motion in MD. In quantum mechanics, the constant of the 


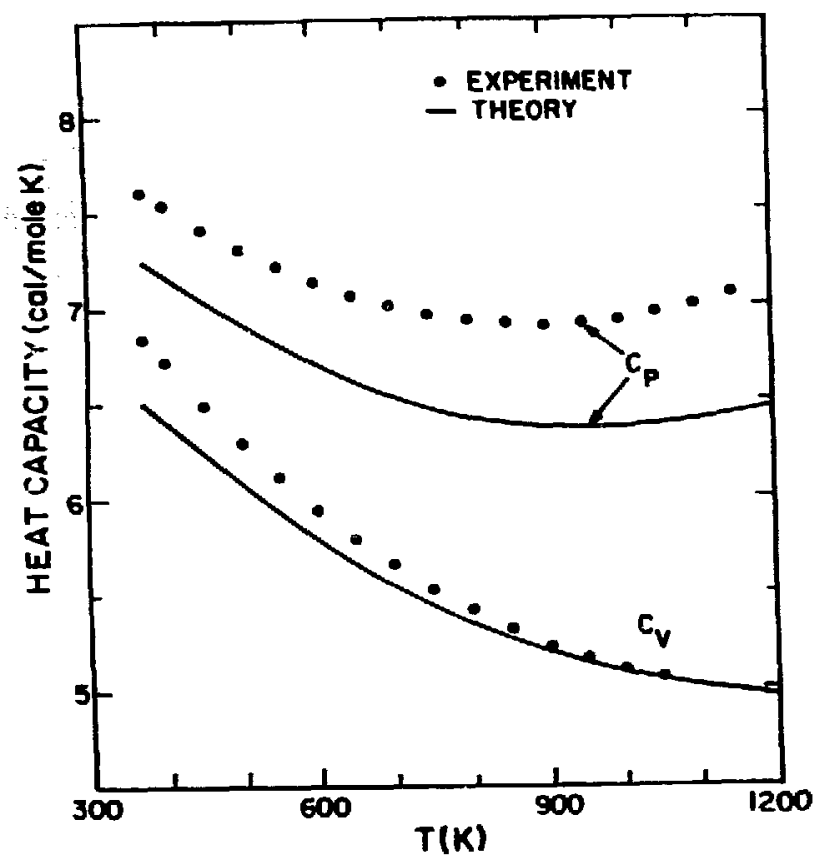

Fig. 10. HEAT CAPACITY AT CONSTANT PRESSURE $\mathrm{C}_{\mathbf{P}}$ AND CONSTANT VOLUME $C_{V}$ IN METALLIC FLUID SODIUM. The solid lines are calculated from MD simulations in the fluid state.

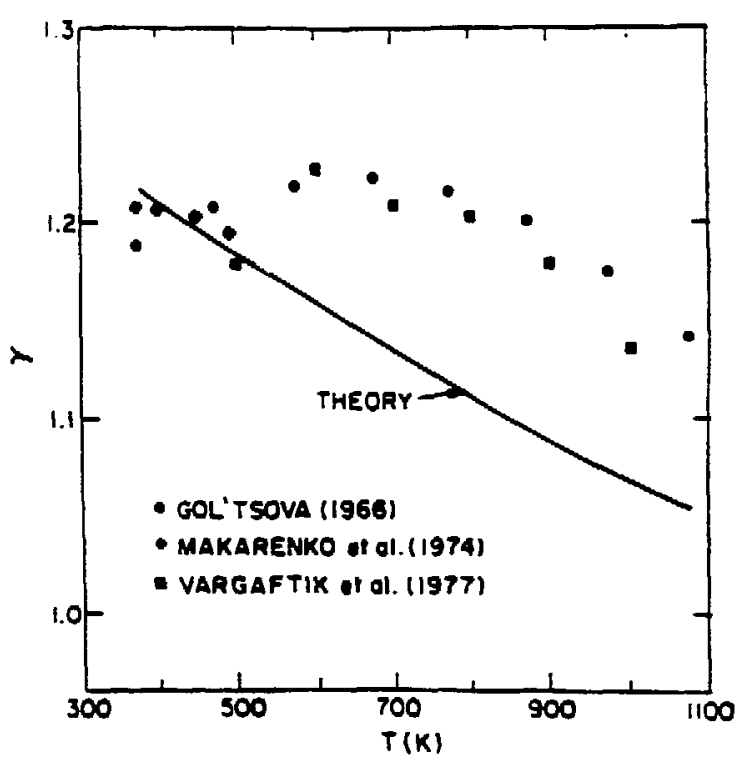

Fig. 11 .

THERMODYNAMIC GRÜNEISEN PARAMETER $\gamma$ VS TEMPERATURE IN METALLIC FLUID SODIUM. The solid lines are calculated from MD simulations in the fluid state and the experimental points were obtained from $\gamma_{\exp }-\mathrm{v} \beta \mathrm{B}_{\mathrm{T}} / \mathrm{C}_{\mathrm{V}}$.

entropy is uniquely determined. (15) We handle the problem in the fluid by making a high temperature cluster expansion of the free energy. If we are able to evaluate $F=F(V, T)$ at any particular point in phase space, then we have determined the entropy constant contained in F=U-TS, implicitly. The constant in the entropy is universal throughout the fluid phase.

The high temperature cluster expansion for a metal contains terms that result from the direct dependence of the potential upon the volume. From the classical partition function for a fluid based on the potential given in Eq.(II.1), the cluster expansion of the Helmholtz free energy written in reduced form $F^{*}$ is $(19)$

$$
\begin{gathered}
F^{*}=\left(F-I_{z}-\Omega\right) / k T=\ln \left[\Lambda^{3} / N\right]-1+\sum_{n=2}^{\infty} B_{n} \rho^{n} / n-1 \\
=\ln \left[\Lambda^{3} / V\right]-1+B_{2} / V+\cdots
\end{gathered}
$$

where $\rho=N / N$ is the atomic density and $\Lambda^{2}=\left(2 \pi \hbar^{2} / \mathrm{mkT}\right)^{1 / 2}$ is the 
thermal de Broglie wavelength. The first coefficient of the summation is

$$
B_{2}=2 \pi \int_{0}^{\infty}\left(1-e^{-\beta \phi(r ; V)}\right) r^{2} d r \text {, }
$$

where $\beta=1 / k T$. In general, $B_{n}=B_{n}(T, V)$. The reduced internal energy is

$$
U^{*}-\left(U-I_{z}-\Omega\right) / k T=3 / 2-T / N \partial B_{2} / \partial T+\cdots .
$$

The reduced pressure is

$$
\mathrm{P}^{*}=(\mathrm{PV}-\mathrm{d} \Omega / \mathrm{dlnV}) / \mathrm{kT}=1+\left(\mathrm{B}_{2} / \mathrm{V}-\partial \mathrm{B}_{2} / \partial \mathrm{V}\right)+\cdots \cdot
$$

We may evaluate $B_{2}$ by a direct integration of Eq. (V.17). If we go to a sufficiently high temperature, then the expansions truncated at $n=2$ will be converged and give an accurate description.

MD again plays a vital role by determining the convergence of the series. MD calculations of $U$ and $P$ as a function of $T$ were carried out for a fixed volume. The MD results are then compared with the second-order cluster. expansions for $\mathrm{D}^{*}$ and $\mathrm{P}^{*}$. It is seen that the approximate series becomes more accurate as the temperature increases, as would be expected. The same behavior is assumed for $F^{*}$. Thus, at a very high temperature (kT-1.16 Ry for $\mathrm{Na}$ ) we may use Eq.(V.16) to evaluate $F^{*}$ which gives us the result that we want, namely, the implicit determination of the constant of the entropy contained in $F^{*}$. The temperature at which the second-order expansions converge is not and need not be a physically meaningful one. The procedure is to evaluate a constant of the entire fluid phase and the trick we have used to accomplish this is theoretically justifiable. (See the discussion of this point in Ref. (20)).

The next step is to integrate down in temperature at constant volume to regions of interest. This process is illustrated in Fig. 12. From the cluster expansion we have evaluated the free energy at the point marked $F^{*}$ High $T$. Using the same MD results in the form of numerical fits of $U_{M D}$, we calculate $U^{*}$ and integrate down in temperature at constant volume with $\mathrm{dF}^{*}=-\mathrm{U}^{\star} \mathrm{d}(\ln \mathrm{T})$.

From this equation the free energy along any line of constant volume may be determined. To obtain the free energy at some other volume, we may fix the temperature and integrate with respect to volume from the equation $\mathrm{dF}^{*}-\mathrm{P}^{*} \mathrm{~d}(1 \mathrm{nV})$.

$\mathrm{P}^{*}$ is also determined from fits to $\mathrm{P}_{\mathrm{MD}}$. Continuing in this manner $F$ may be calculated for all volumes where MD runs have been made. 


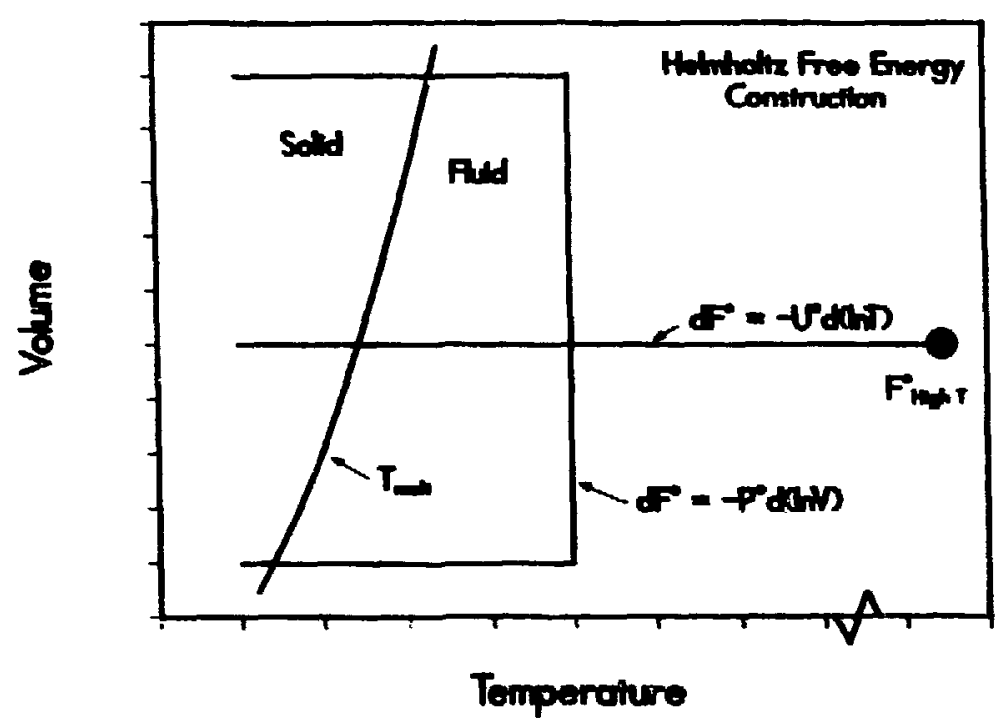

Fig. 12 .

DETERMINATION OF THE ENTROPY CONSTANT FOR THE FLUID PHASE AND CONSTRUCTION OF THE EQUILIBRIUM MELT LINE FROM THE GIBBS FREE ENERGY. The free energy is evaluated at a temperature $\mathrm{T}_{\mathrm{High}}$ using the cluster expansion of $\mathrm{Eq}$. (V.16) to second order and using MD to determine the convergence of the expansion. This implicitly determines the constant of entropy contained in $F$ for the entire fluid phase. The free energy can then be constructed throughout the phase using the $M D$ results for $U^{\star}$ to integrate along lines of constant volume and MD results for $\mathrm{P}^{\star}$ for lines of constant temperature.

\section{G. Solid-Fluid Phase Boundary}

Having constructed the free energies in both the solid and fluid phases, we may establish the phase boundary for equilibrium melting. With the Gibbs function $G=F+P V$, and the subscripts $f$ or $s$ for fluid or solid, respectively, the fluid-solid coexistence equations are

$$
\begin{aligned}
& P_{f}\left(V_{f}, T_{m}\right)=P_{s}\left(V_{s}, T_{m}\right)=P_{m}\left(T_{m}\right), \\
& G_{f}\left(P_{m}, T_{m}\right)-G_{s}\left(P_{m}, T_{m}\right) .
\end{aligned}
$$

These equations were solved at each of the volumes of the $M D$ runs for $T_{m}$ and $P_{m}$. The dashed line marked "Theory" in Fig. 13 is the result. The solid line is the experimental melting curve. The solid squares are the MD results at only one of the volumes, $V=256 \mathrm{a}_{0}^{3}$; dynamic melting takes place between the highest point on the solid-phase branch $(385 \mathrm{~K})$ and the lowest point on the fluid-phase branch $(396 \mathrm{~K})$. The agreement with experiment is excellent and further comparisons are made in Ref. (20).

As a final point, the question of the relative importance of the volume 


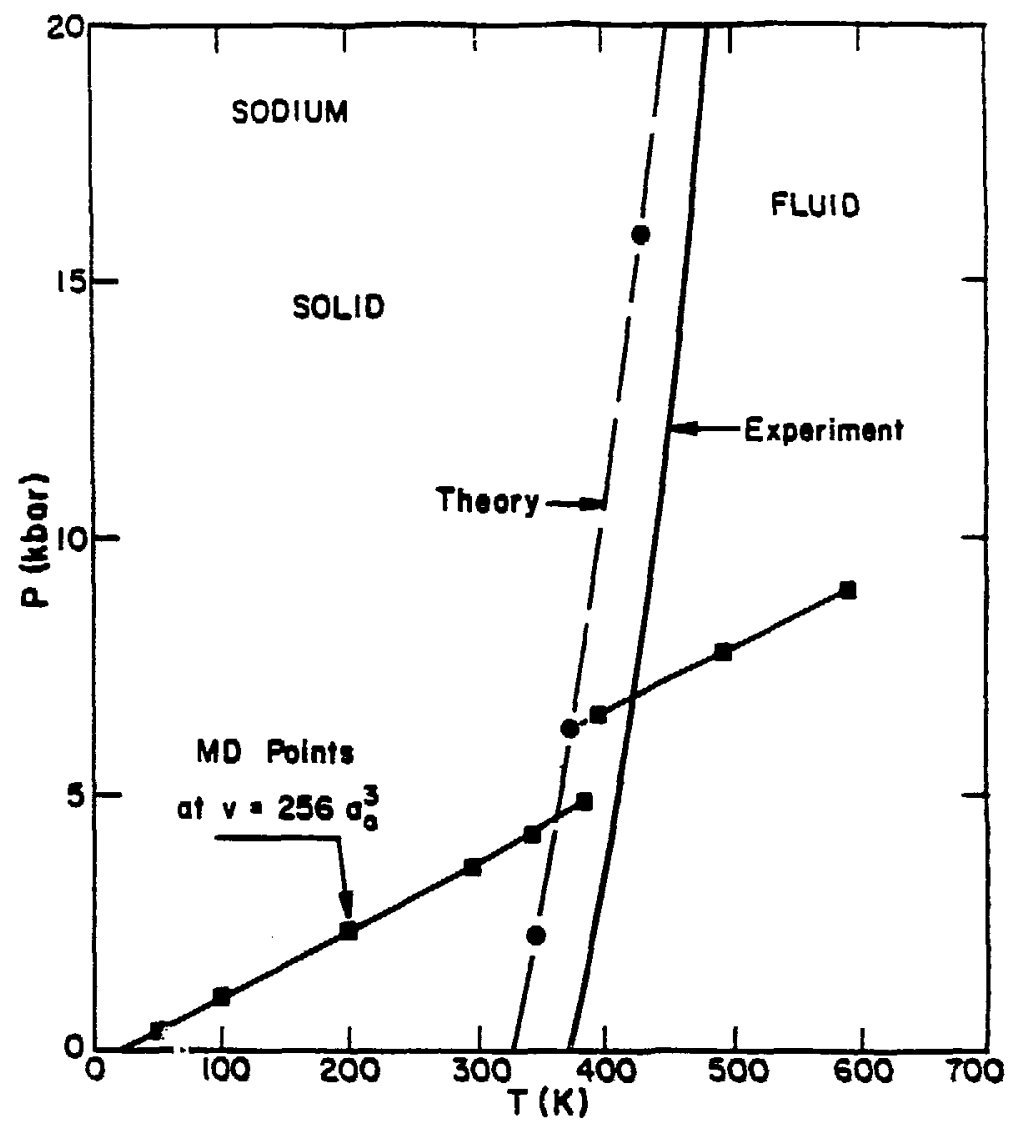

Fig. 13.

P-T PHASE DIAGRAM FOR SODIUM. The equilibrium melting line is the result of solving for $P_{m}$ and $T_{m}$ as given in Section V.G. The solid line is the experimental melting curve, and the solid circles show the calculated equilibrium melting points. The solid squares are MD results for the fixed volume of $256 \mathrm{a}_{0}^{3}$ /atom; dynamic melting takes place between the highest point on the solid-phase branch $(385 \mathrm{~K})$ and the lowest point on the fluid-phase branch (396 K).

terms $\Omega(V)$ to $\sum \phi$ can be examined. When sodium melts at zero pressure, there is a 2.48 change in volume with a principal contribution coming from $\Omega(V)$. Denoting the change in a quantity upon melting by $\Delta$, we see from our calculations:

$\Delta \Omega=296 \mathrm{cal} / \mathrm{mole}, \Delta \sum \phi=252 \mathrm{cal} / \mathrm{mole}, \Delta \mathrm{U}_{\text {total }}=548 \mathrm{cal} / \mathrm{mole}$.

The last number may be compared with $\Delta \mathrm{U}_{\mathrm{exp}}=621 \mathrm{cal} / \mathrm{mole}$. Thus, we see that the volume dependent terms account for more than haif of the change in the internal energy upon melting and are therefore essential. 
The theory of quasiharmonic lattice dynamics has been extremely successful for the calculation and interpretation of the thermodynamic properties of insulators, semiconductors, metals and compounds. In this Section, we will sketch the results from lattice dynamics necessary to provide a comprehensive analysis of MD si nulations. We will follow closely the presentation of Wallace in Thermodynamics of Crystals ${ }^{(5)}$ where the theory is developed in detail and applied to insulators and metals. We will present the formulas necessary for the evaluation of the phonon frequencies using the effective two-body potential of Section III and give the results for $\mathrm{Ni}$ and $\mathrm{Cu}$.

The theory of lattice dynamics is a fully quantum mechanical theory treating a system of electrons and ions. One can write the total crystalline Hamiltonian in the form $H=K . E .+\Phi$ where K.E. is the kinetic energy of the ions and $\Phi$ is the total adiabatic potential in the Born-oppenheimer approximation and contains the kinetic energy of the electrons and the potential energy of the interacting ions electron system. One then expands $\Phi$ about the equilibrium positions (crystalline lattice sites); for the harmonic theory one includes terms to second order in the displacements where higher order terms may be included in perturbation theory. The quasiharmonic Hamiltonian is $H=\Phi_{0}+\mathrm{H}_{2}$. Now $\Phi_{0}$ is the adiabatic potential evaluated at the equilibrium positions of the ions and includes the entire volume dependence of the metallic potential as discussed in Sec.II.

We solve $\mathrm{H}_{2}$ for its normal vibrational modes (phonons). In matrix notation the eigenvalue-eigenvector equation is

$$
\vec{v}^{*}(\vec{k}) \cdot D(\vec{k}) \cdot \vec{v}(\vec{k})=\vec{\omega}^{2}(\vec{k}) \text {. }
$$

This set of equations is solved for the vibrational frequencies at each wavenumber $\vec{k}$. The $\vec{v}(\vec{k})^{\prime}$ s are the polarization vectors that diagonalize $D(\vec{k})$ and give the set of ionic displacements for a particular mode $\vec{k}$. $D$ is the dynamical matrix

$$
D_{i j}(\vec{k})=1 / M \sum^{\prime} n \Phi_{i j}\left(r_{n}\right)\left(\cos \vec{k} \cdot \vec{r}_{n}-1\right),
$$

where $M$ is the ion mass, the summation is over all lattice sites (the prime means to exclude the term for $r_{n}=0$ ), and $i, j$ are indices for the cartesian coordinates $x, y, z$. The potential energy coefficients are the second-order derivatives of the adiabatic potential with respect to the displacements and are 
given by

$$
\Phi_{i j}-\partial^{2} \Phi / \partial u_{i} \partial u_{j}
$$

with the cartesian components of the displacements $u_{i}=r_{i}-r_{i 0}, r_{i 0}=$ cartesian component of the lattice positions. In terms of the $\phi(r ; V)$ of section 3 ,

$$
D_{i j}(\vec{k})=1 / M \sum_{n}^{\prime}\left\{2 \phi^{\prime}\left(r_{n}^{2}\right) \delta_{i j}+4 \phi^{\prime \prime}\left(r_{n}^{2}\right) r_{n i} r_{n j}\right\}\left(1-\cos \vec{k} \cdot r_{n}\right) \text {. }
$$

$\delta_{i j}-1$ for $i-j$ and 0 for $i \neq j$. The primed quantities are defined to be derivatives of the two-body potential with respect to $r^{2}$ :

$$
\phi=\phi\left(r^{2}\right) ; \phi^{\prime}=\partial \phi / \partial\left(r^{2}\right) ; \quad \phi^{\prime \prime}=\partial^{2} \phi / \partial\left(r^{2}\right)^{2} .
$$

For the Wills-Harrison potential in the transition metal series

$$
\phi=\mathrm{v}_{\mathrm{F}}+\mathrm{v}_{\mathrm{B}}+\mathrm{v}_{\mathrm{C}} \text {, }
$$

as given by Eqs. (III.2)-(III.4), and

$$
\begin{aligned}
& \phi^{\prime}=-V_{F}(1 / r+\kappa) / 2 r+V_{B}^{\prime}+V_{C}^{\prime} \text {, } \\
& \phi^{\prime \prime}=\mathrm{v}_{F}\left(\kappa^{2} \mathrm{r}^{2}+3 \kappa \mathrm{r}+3\right) / 4 \mathrm{r}^{4}+\mathrm{V}_{\mathrm{B}}{ }^{\prime}+\mathrm{V}_{\mathrm{C}}{ }^{\prime} \text {, } \\
& \mathrm{V}_{\mathrm{B}}{ }^{\prime}=-5 \mathrm{~V}_{\mathrm{B}} / 2 \mathrm{r}^{2} \quad, \quad \mathrm{~V}_{\mathrm{B}}{ }^{\prime}=35 \mathrm{~V}_{\mathrm{B}} / 4 \mathrm{r}^{4} \text {, } \\
& \mathrm{v}_{\mathrm{C}^{\prime}}=-4 \mathrm{~V}_{\mathrm{C}} / \mathrm{r}^{2} \quad, \quad \mathrm{~V}_{\mathrm{C}^{\prime}}{ }^{\prime}-20 \mathrm{v}_{\mathrm{C}} / \mathrm{r}^{2} \text {. }
\end{aligned}
$$

The contributions from the d-electron repulsion $V_{C}$ and the formation of bands $v_{B}$ are to be included with the conduction electron potential $v_{F}$.

Eq. (VI.1) is solved by making Fourier expansions of the displacements $u$, leading to the phonon coordinates $q$. The $q^{\prime} s$ constitute a complete set of $3 \mathrm{~N}$ coordinates (one atom per unit cell) capable of a complete description of the vibrational motion. The set of $3 \mathrm{~N}$ allowable wavenumbers $\vec{k}$ is determined by constructing the Brillouin zone in the reciprocal lattice and contains the full symmetry properties of the crystal. The reader unfamiliar with the Brillouin zone terminology is referred to Ref.(5), p.105ff. It is interesting to note that any potential function that gives all $3 \mathrm{~N}$ phonon eigenvalues is the potential and completely describes the system. In practice we only know the phonons along the principal symmetry directions from experiment.

\section{A. Dispersion Curves for Nickel and Copper}

In Fig. 14 the phonon dispersion curves are plotted for nickel and copper, respectively, using the experimental data of Birgeneau ${ }^{21}$ and Svensson et al. 22 The best overall fit was determined by adjusting $r_{c}$ while leaving $r_{d}$ fixed. $r_{d}$ is determined from first-principles ${ }^{13}$ and within the framework of the theory, it is not a free parameter. The overall agreement with experiment is quite excellent although the calculated phonon frequencies $\nu$ for the transverse 
68 decrease in the average of all the phonon frequencies. If one neglects the effects of the d-electrons, then the overall agreement is poorer.

\section{B. Phonon Thermodynamics}

The Helmholtz free energy for the phonons in the harmonic approximation is $\mathrm{F}_{\mathrm{H}^{-}} \sum_{\mathrm{k}}\left\{1 / 2 \hbar \omega(\overrightarrow{\mathrm{k}})+\mathrm{kT} \ln \left(1-\mathrm{e}^{-\hbar \omega(\overrightarrow{\mathrm{k}}) / \mathrm{kT}}\right)\right\}$,

and the first term is the zero-point vibrational energy. The internal energy, pressure, entropy, heat capacity, etc. are defined by the appropriate derivatives of $\mathrm{F}$ as discussed in Sec. IV although here the description is quantum mechanical and contains the correct behavior at low temperatures, for instance, of the heat capacity as shown in Fig. 8. The connection between harmonic phonon theory and the classical MD simulations is made by taking the high temperature limit of the harmonic free energy given by

$$
F_{H^{-}}-k T \sum_{k}\left(\ln (k T / \hbar \omega(\vec{k}))-1 / 24(\hbar \omega(\vec{k}) / k T)^{2}+\cdots\right\} \text {. }
$$

In this limit, we need to evaluate

$$
\left.\langle\operatorname{lnk} \theta\rangle_{-}<\ln \hbar \omega\right\rangle_{\mathrm{B}} . \mathrm{z} .-1 / 3 \mathrm{~N} \sum_{\mathrm{k}} \ln \hbar \omega(\overrightarrow{\mathrm{k}}) \text {. }
$$

The summation is over the $3 \mathrm{~N}$ values of $\vec{k}$ and this average may be evaluated for a given Brillouin zone using the technique in Sec. 37 of Ref. (5). This is the quantity needed in Section V.B to analyze the MD simulations. Since we also need volume derivatives of <lnkes, a linear numerical fit as a function of $V$ of Eq. (B.13) was made and determined to be adequate.

\section{ACKNOWLEDGEMENTS}

It is a pleasure to acknowledge useful conversations with Dr. D. C. Wallace and Professor J. R. Beeler. The figures were prepared with the capable assistance of Dr. M. C. Brady. 

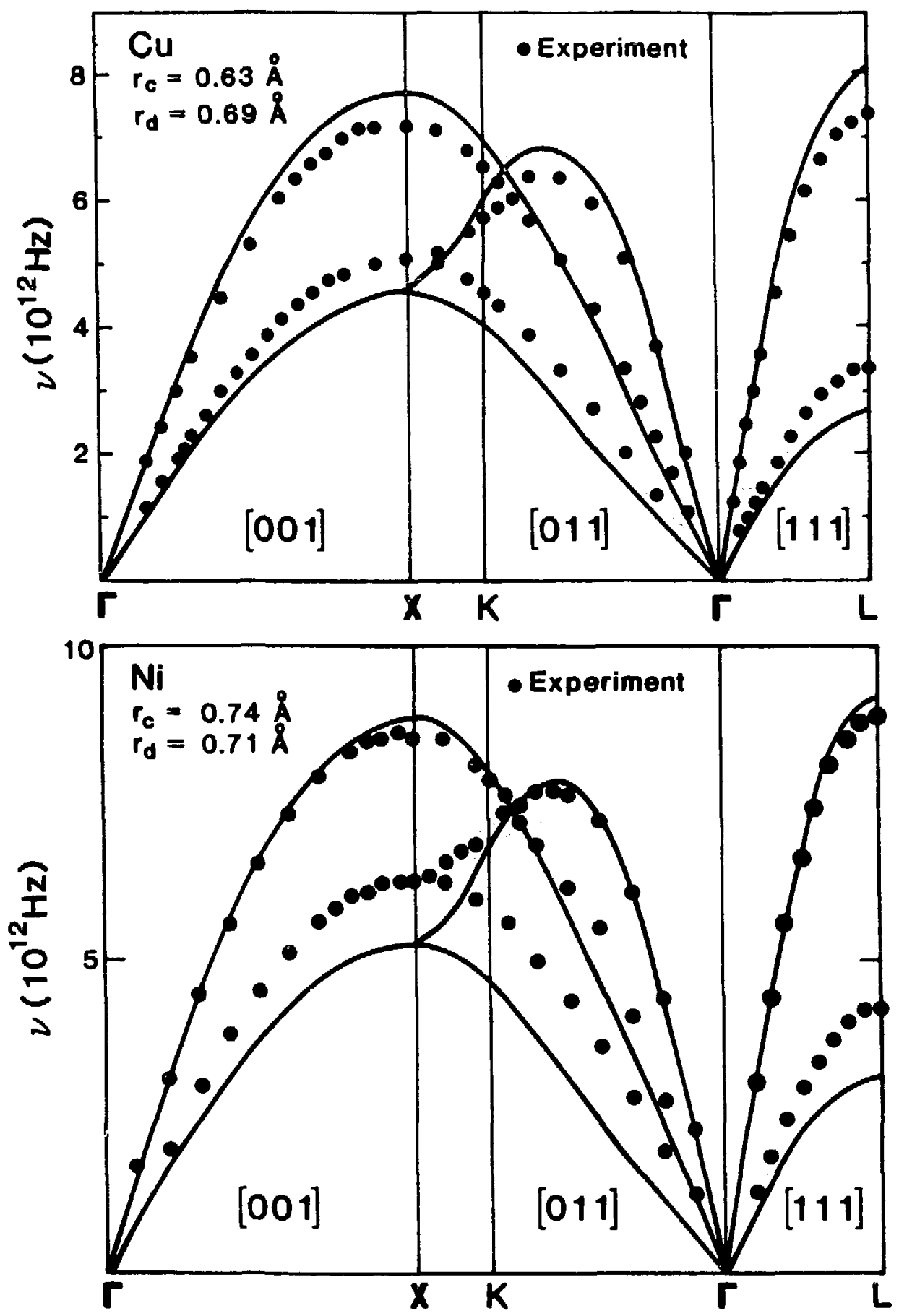

Fig. 14

PHONON DISPERSION CURVES FOR COPPER AND NICKEL. The value of the empty-core pseudopotential parameter $r_{c}$ was adjusted for the best overall agreement with experiment. 


\section{REFERENCES}

1. D. C. Wallace and G. K. Straub, Phys. Rev. A27, 2201 (1983).

2. D. C. Wallace, in Electronic Structure, Dynamics, and Quantum Structural Properties of Condensed Matter, ed. by J. T. Devreese and Piet Van Camp (Plenum Publishing Corporation, 1985), p. 521.

3. D. C. Wallace, S. K. Schifer1, and G. K. Straub, Phys. Rev. A. 30,616 (1984).

4. S. K. Schiferl and D. C. Wallace, Phys. Rev. B 31,7662 (1985).

5. D. G. Wallace, Thermodynamics of Crystals (Wiley, New York, 1972).

6. Ref. 5, Chapter 3 and Appendix B.

7. W. A. Harrison, Pseudopotentials In The Theory of Metals (Benjamin, New York, 1966).

8. W. A. Harrison, Electronic Structure and the Properties of Solids

(Freeman, San Francisco, 1980).

9. R. E. Swanson, G. K. Straub, B. L. Holian, and D. C. Hallace, Phys. Rev. B27, 2873 (1983).

10. G. K. Straub, R. E. Swanson, B. L. Holian, and D. C. Wallace, in Ab Initio Calculation of Phonon Spectra, ed. by J. T. Devreese, V. E. Van Doren and P. E. Van Camp (Plenum, New York, 1983), p. 137.

11. J. M. Wills and W. A. Harrison, Phys. Rev. B28, 4363 (1983).

12. W. A. Harrison and J. M. Wills, Phys. Rev. B25, 5007 (1982).

13. G. K. Straub and W. A. Harrison, Phys. Rev. B31, 7668 (1985).

14. S. K. Schiferl and D. C. Wallace, J. Chem. Phys. 83, 5203 (1985).

15. F. Reif, Fundamentals of Statistical and Thermal Physics (McGraw-Hill, New York, 1965).

16. The term "exact" refers to a full non-1inear solution to the coupled many-body equations of motion limited only by the size of the computer. For a constant volume calculation, the total energy may be conserved to the accuracy of the computer by making the time step small enough; this is a practical limit but is not a significant problem. We must worry also about the accuracy of $\Phi$; this must ultimately be resolved by comparison with experiment.

17. G. K. Straub, S. K. Schifer1, and D. G. Wallace, Phys. Rev. B28, 312 (1983).

18. G. K. Straub and D. C. Wallace, Phys. Rev. B $\underline{30}, 3929$ (1984). 
19. D. C. Wallace, B. L. Holian, J. D. Johnson, and G. K. Straub, Phys. Rev. A 26, 2882 (1982).

20. B. L. Holian, G. K. Straub, R. E. Swanson, and D. C. Wallace, Phys. Rev. B $\underline{25}, 7807$ (1982).

21. R. J. Birgeneau, Phys. Rev. 136 , A1359 (1964).

22. E. C. Svensson, B. N. Brockhouse, and J. M. Rowe, Phys. Rev. 155, 619 (1967). 